\title{
sensors
}

ISSN 1424-8220

(C) 2007 by MDPI

www.mdpi.org/sensors

Review

\section{Optical Fiber Sensing Using Quantum Dots}

\author{
Pedro Jorge ${ }^{1, *}$, Manuel António Martins ${ }^{2}$, Tito Trindade ${ }^{2}$, José Luís Santos ${ }^{1,3}$ and Faramarz \\ Farahi $^{4}$
}

1 Unidade de Optoelectrónica, INESC Porto. Rua do Campo Alegre, 687. 4169007 Porto, Portugal. e-mail: pjorge@inescporto.pt. e-mail: jlsantos@inescporto.pt

2 Department of Chemistry - CICECO, University of Aveiro. 3810-193 Aveiro, Portugal e-mail: mamartins@ua.pt.e-mail: tito@ua.pt

3 Dept. Física. Faculdade de Ciências, Universidade do Porto. Rua do Campo Alegre, 687. 4169007 Porto, Portugal.

4 Department of Physics \& Optical Science. UNC Charlotte, 306-A Grigg. Charlotte, NC 28223-0001 e-mail: ffarahi@uncc.edu

* Author to whom correspondence should be addressed.

Received: 19 November 2007 / Accepted: 20 December 2007 / Published: 21 December 2007

\begin{abstract}
Recent advances in the application of semiconductor nanocrystals, or quantum dots, as biochemical sensors are reviewed. Quantum dots have unique optical properties that make them promising alternatives to traditional dyes in many luminescence based bioanalytical techniques. An overview of the more relevant progresses in the application of quantum dots as biochemical probes is addressed. Special focus will be given to configurations where the sensing dots are incorporated in solid membranes and immobilized in optical fibers or planar waveguide platforms.
\end{abstract}

Keywords: quantum dots, biochemical sensors, optical fibers.

\section{Introduction}

The advent of Nanotechnology, introducing control over matter at the nanometer scale, has produced a new class of materials with novel properties, creating new possibilities in a diversity of domains. In particular, biotechnology is already taking advantage from the versatility of nanoparticles with a variety of sizes, shapes and compositions (metal, polymer, semiconductor,...) [1-3]. The 
reduced dimensions of the particles is a key factor leading to the possibility of enhancement and tailoring of many of the material properties (electrical, optical, chemical...) which, at such scale, become size and/or shape dependent. Enhanced mechanical properties, tunable light scattering or luminescence due to quantum size effects, are examples of features that can be controlled at the nanoscale. In addition, the small size of the particles provides a large interfacial area which enables bioconjugation (i.e., combination of the nanoparticle with biomolecules like antibodies) allowing such properties to be integrated in biological systems. In this way, the performance of conventional techniques can be largely improved and an entirely new set of exciting applications becomes available [4-6].

In this context, luminescent semiconductor nanocrystals, or quantum dots (QDs), are particularly attractive for biochemical sensing and imaging applications. Fluorescence is the basis of a large number of bioassays and chemical sensing techniques. In this regard, the unique optical properties of QDs are highly favorable when compared to those of traditional molecular fluorophores. The ability to tune their luminescence characteristics by particle size control, combined with relatively high quantum yields, narrow fluorescence emission, very broad absorption spectrum and an outstanding photostability provide new solutions to many of the problems associated with traditional luminescence sensors and are the promise for a completely new set of applications [7-9].

QDs' unique features have attracted a considerable interest and the variety of new applications is expanding quickly. Although this is a relatively recent field of research, the synthesis of QDs associated to their applications in a diversity of domains has been the subject of several reviews. Early reviews focused on synthesis, functionalization and material properties, and provided a prospective view of the field $[10,11]$. More recently, the use of QDs as labels in biomedical imaging or in immunoassays has seen several breakthroughs which were analyzed by different authors [8, 12, 13]. Very good reviews have been published on the use of QDs in new sensing strategies, giving either a general overview of the field [14], or a more focused discussion on biosensing applications and future trends [15-17]. Other fields of interest such as laser applications or fundamental physical experiments have also been reviewed [18-20].

Nevertheless, due to the large research efforts dedicated to this topic, a number of sensing applications has recently appeared and this is currently an expanding field of research. In particular, the immobilization of nanocrystals in solid membranes and its use in combination with optical fiber or planar platforms has seen some progress. Such arrangements are a key step towards the development of advanced analytical instrumentation, aiming small scale and multiparameter capability. For this, QDs are very well suited because of their high photostability and multiplexing ability.

In this paper, a brief overview on recent progress in QDs sensing applications is described. Particular focus will be given to an emerging area: the application of QDs of II/VI materials as sensing elements while immobilized in a solid host which can be used in combination with optical fiber or integrated optics sensing technology. 


\section{Quantum dots: a brief overview}

Quantum Dots are small particles of a semiconductor material, consisting of a few hundreds to thousands of atoms. Their small size, ranging for most of the systems from $1 \mathrm{~nm}$ to $10 \mathrm{~nm}$, is mostly responsible for their unique optical, electrical and chemical properties.

The main procedures by which QDs can be fabricated, attaining 3D confinement of the charge carriers, include diffusion controlled growth, lithography, epitaxy and colloidal chemistry. Combined lithographic patterning and etching is a possible pathway [21], and epitaxial growth over pre-patterned substrates has also been investigated [22]. More recently, techniques exploring self-assembly mechanisms are being successfully explored [20]. Nevertheless they rely on expensive MBE or MOCVD systems. In all physical deposition approaches the resulting QDs are embedded in a solid matrix and are, therefore, more adapted to integrated optoelectronic devices (QD lasers and detectors). Other techniques have been explored for applications requiring further chemical manipulation and processing.

Alternative approaches include the synthesis of QDs using colloidal chemistry techniques which are very often associated to molecular precursor chemistry. For these methods the semiconductor nanoparticles are homogeneously generated in a coordinating solvent or in the presence of a chemical stabilizer. The synthesis of QDs in high boiling point solvents have been particularly successful in yielding nearly monodispersed QDs with very narrow emission bands [10, 18]. Because QDs produced in this way have their surfaces capped with organic ligands, they are compatible with further (bio)chemical surface modification. So, they are particularly suited for sensing applications involving luminescence. This review will focus on the sensing applications of QD-based devices.

\subsection{Quantum confinement and optical properties}

The main differences between the macrocrystalline semiconductor and the corresponding nanocrystalline material arise from two fundamental factors that are size related. The first one is associated to the large surface area to volume ratio of nanoparticles and the second one is related to the three-dimensional quantum confinement of their charge carriers. In the particular case of semiconductors, quantum confinement takes place whenever the nanoparticles' size is smaller than the exciton Bohr radius of the bulk semiconductor, $a_{\mathrm{B}}$ (typically in the $1 \mathrm{~nm}$ to $10 \mathrm{~nm}$ range which is still much larger than the semiconductor lattice constant, $<1 \mathrm{~nm})$.

A direct consequence of the $3 \mathrm{D}$ confinement is that the energy levels of the excited carriers (exciton) will become discrete and approach the molecular behavior as the particle size decreases. An ideal quantum dot can be treated like a spherical quantum box, and will display an atomic like absorption spectrum. The energies of the quantized states in the conduction and valence "band" can be calculated using the Schrödinger equation and the effective mass approximation. However, considering that both electron and hole are confined into a space smaller than the Bohr radius of the exciton, they cannot be considered as mutually independent making the solutions of the equation harder to get. Many authors have suggested different approaches to this problem from which resulted some approximate analytical expressions or even numerical solutions that are in good agreement with experimental data [23]. Brus et al. [24] showed that, for cadmium sulfide (CdS) and cadmium selenide 
(CdSe) nanocrystals, the size dependence for the fundamental electron-hole state, $E_{1 \mathrm{~S} 1 \mathrm{~s}}$, can be described by

$$
E_{1 S 1 s}=E_{g}+\frac{\pi^{2} h^{2}}{2 a^{2} \mu}-1.786 \frac{e^{2}}{\varepsilon a},
$$

where $a$ is the particle radius, $\mu$ the electron-hole reduced mass, $e$ the electronic charge and $\varepsilon$ the dielectric constant of the bulk semiconductor. The first term on the right, $E g$, corresponds to the bulk bandgap energy, the second term accounts for the confinement energy and the third term for the electron-hole Coulomb interaction. Equation (1) shows that, besides inducing energy quantization, decreasing the dots size makes the Coulomb term shift the total energy to lower values with a $a^{-1}$ dependence. Conversely, the confinement term adds to the total energy with a $a^{-2}$ dependence. This way, for smaller dot sizes, the confinement term becomes dominant and the optical spectrum shows a blue shift in the band edge energy when the QD's size is decreased below $a_{B}$. The smaller the dot the greater will be the blue shift observed relative to the typical $E_{g}$ of the bulk semiconductor.

Like in bulk semiconductors, the band edge energy determines the absorption onset and the luminescence peak emission (near band edge). Therefore, QDs will absorb any photon with energy $h v>\mathrm{E}_{1 \mathrm{~S} 1 \mathrm{~s}}$ and display an emission peak around the same energy value. In this context, Equation (1) shows that QD technology allows band gap tuning by control of the nanoparticles size $(a)$ or material type $(\varepsilon)$.

As the nanoparticles size decreases, an increasing overlap of the confined charge carriers wave functions translates into strongly enhanced absorption coefficients. Because the amount of possible transitions grows with photon energy, the absorption coefficient increases steadily as the excitation wavelength is shifted towards the blue. On the other hand, emission processes in QDs result from electron-hole recombination and are strongly dependent on the competition between such radiative processes and non-radiative recombination mechanisms. Non-radiative processes occur mainly at defects located at the nanocrystal surface. In this context, the large surface/volume ratio of QDs allows to obtain enhanced quantum yields by control of their surface chemistry and passivation of surface defects. In a particularly successful strategy, overcoating the nanocrystal core $(\mathrm{CdSe})$ with an outer shell of an higher bandgap semiconductor $(\mathrm{ZnS})$ resulted in quantum yields in the $50 \%$ range. Besides the increased brightness, core-shell systems provide increased photostability and chemical resistance $[25,26]$. More recently, highly luminescent CdSe core nanocrystals caped with a multi-shell layer (CdS and $\mathrm{ZnS}$ ) have been reported, displaying quantum yields in a 70-85 \% range [27].

While an ideal quantum dot displays an atomic like discrete spectrum, in synthetic nanocrystals the linewidths are limited by thermal broadening. This way, the emission spectrum of a single QD can have, at room temperature, full widths at half maximum (FWHM) of a few nanometers. However, the major source of emission broadening in QDs comes from their size distribution. In a colloidal dispersion the solid particles have approximately, but not exactly, the same size. Because the emission of a QD is related to its size, the slight differences in size result in slight variations in the emission wavelength. As a consequence, the emission spectrum of a certain nanocrystal ensemble will be much broader than the individual QDs spectra. Currently, it is possible to achieve size distributions with 
variation lower than 5\%. This translates into a FWHM of approximately 25-30 nm, which is quite narrow in comparison to the spectral response of many luminescent dyes.

A typical absorption spectrum of a CdSe QD ensemble is shown in Figure 1 (left). In contrast with traditional dyes, which have broad emission spectra with a characteristic long red tail, nanocrystals present a symmetrical (approximately Gaussian) and relatively narrow emission profile and a very broad absorption spectrum. These two features combined allow to obtain a large equivalent Stokes shift, which can be tuned by setting excitation at any wavelength lower than the emission peak, facilitating the discrimination of the QDs' luminescent emission. In addition, it also introduces the possibility of exciting different QDs with the same optical source. This can be observed in Figure 1 (right) where the emission of different CdSe and CdTe QDs, coated with a ZnS shell, was obtained by excitation with a single blue LED.
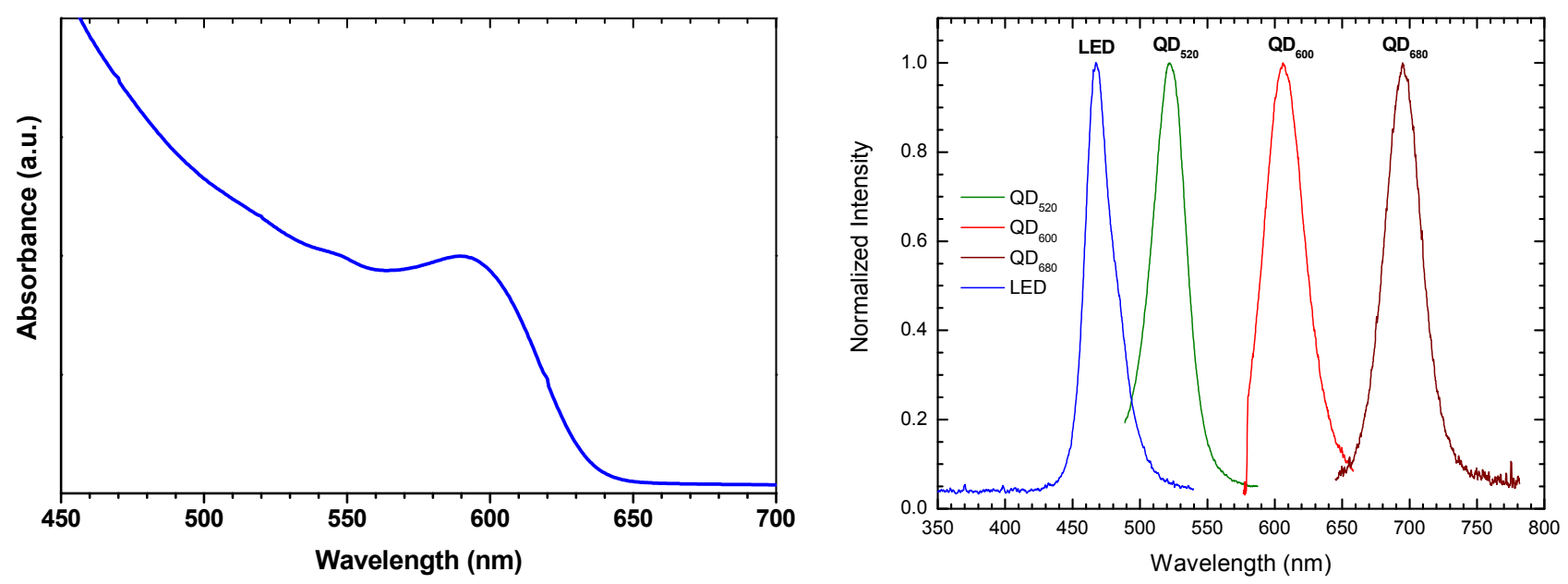

Figure 1. Left -Typical absorption spectrum of CdSe nanocrystal QDs. Right - Normalized emission spectra of different samples of core-shell QDs immobilized in a sol-gel matrix. Peak emissions from blue to red are $520 \mathrm{~nm}(\mathrm{CdSe} / \mathrm{ZnS}), 610 \mathrm{~nm}(\mathrm{CdSe} / \mathrm{ZnS})$ and $680 \mathrm{~nm}(\mathrm{CdTe} / \mathrm{ZnS})$. The spectrum of the excitation source, a blue LED $(470 \mathrm{~nm})$, is also shown.

Depending on the particles size, the emission of CdSe quantum dots can be continuously tuned from $465 \mathrm{~nm}$ to $640 \mathrm{~nm}$, corresponding to a size ranging from $1.9 \mathrm{~nm}$ to $6.7 \mathrm{~nm}$ (diameter), respectively. This range can be extended with cadmium-telluride ( $\mathrm{CdTe}$ ) quantum dots which emit further into the red $(600 \mathrm{~nm}-725 \mathrm{~nm})$. Core-shell systems of cadmium-based semiconductor materials overcoated with $\mathrm{ZnS}$, provide bright luminescence over the whole visible range and are the preferred systems of most sensing applications. Figure 2 shows typical samples of organically capped CdSe/ZnS QDs, dispersed in toluene, under UV irradiation at room temperature. Higher-energy emission is possible with $\mathrm{CdS}$ $(350 \mathrm{~nm}-470 \mathrm{~nm})$. Infrared emission is also available (780nm-2000nm) using indium arsenide (InAs), or lead selenide /sulfide $(\mathrm{PbSe} / \mathrm{PbS})$ nanocrystals.

In comparison with conventional molecular dyes, QDs have bright, narrow and stable photoluminescence, provide larger equivalent Stokes shifts, and allow to tune their properties. This set of characteristics gives nanocrystals a great advantage in many traditional luminescence applications. All these features are highly attractive for optical fiber and planar platforms applications. 
In particular, QDs' wavelength multiplexing ability is very well suited for many existing optical fiber or microarray configurations. In this regard, having identical nanoparticles from the material point of view, but with different optical properties, introduces the possibility of batch processing multiparameter/multipoint sensors with much simpler procedures. Each set of nanoparticles can be prepared with similar quantum yield, surface chemistry and set of environmental sensitivities. Conversely, when using traditional dyes, different emission wavelengths are provided by completely different chemical species, sometimes presenting dramatically different quantum yields and chemical properties.

In fiber and planar waveguide platforms, the increased photostability of core-shell QDs is a key feature. In fact, in such devices sensing can take place at very small scale and small sample volumes can be easily exposed to very high excitation energy densities. While most dyes present severe photodegradation when illuminated by energetic radiation, quantum dots have demonstrated to be photostable in most situations. Although photobleaching has been reported in bare dots, nanocrystals with an adequate protective shell are known to remain extremely bright even after several hours of exposure to moderate to high levels of UV radiation. On the other hand, the luminescence emission of common dyes can vanish completely after a few minutes.

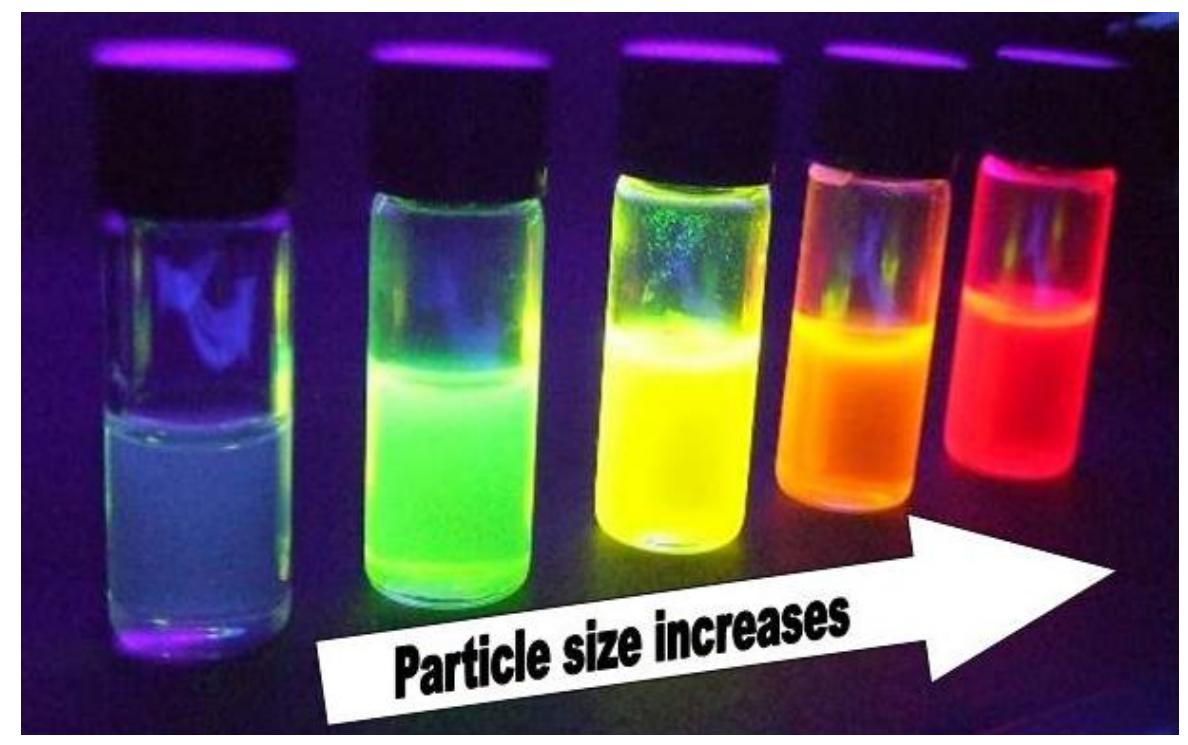

Figure 2. Photoluminescent $\mathrm{CdSe} / \mathrm{ZnS}$ QDs dispersed in toluene (UV light irradiation).

In practice, the photostability of nanocrystals depends on their surface coatings (bare dots, core shell, or other), and on the surrounding environment (solution or solid matrix). Depending on these circumstances, different behaviors have been observed. Some authors reported photo-enhancement, i.e., a gradual increase of the luminescence intensity, under UV irradiation, both in solution and in solid hosts [28, 29]. Both reversible and non-reversible photo-enhancements were observed and, usually, both components can coexist. The increase in luminescence is explained by a light-induced rise of the dots potential barrier, preventing excited carriers from escaping the QDs and favoring radiative recombination. Photo-induced passivation of surface defects is another possible mechanism involved. After enhancement, slow photo-degradation usually follows. The time scales of these behaviors depend strongly on the power densities to which the samples are submitted. 
For CdSe nanocrystals, encapsulated in a glass matrix, Verma and collaborators observed that the photoluminescence intensity showed a linear dependence with the excitation optical power up to moderately high power densities (aprox. $400 \mathrm{~W} / \mathrm{cm}^{2}$ ). For higher excitation powers, excited carrier densities dramatically increased and the observed dependence was no longer linear. In addition, shifts in the band-edge luminescence could be observed either due to 'band-filling' processes (blue shift) or to photo-induced heating of the sample (red shift)[30].

Much research has been carried out regarding QDs' photostability and apparently contradictory results are often reported. However, these apparent contradictions are often caused by differences in host environments and/or distinct nanocrystals surfaces chemistry. While QDs surface chemistry is the key to obtain highly photostable nanocrystals, it is also the way by which the nanoparticles can become sensitive to a given analyte, can be rendered water soluble, or can become biocompatible. This control is typically achieved by functionalizing the dots surface with different chemical or biological ligands and will ultimately determine the usefulness of QDs as a sensing tool.

\subsection{Chemical synthesis}

Long before the popularization of QDs, a theoretical framework concerning QDs' properties had already been established [31]. However, pioneering synthesis methods failed to provide high quality QDs. Arrested precipitation in solution generally yielded QDs with a low degree of crystallinity and broad size distributions, while synthesis in confined structured medium, such as in porous materials, poses problems with the recovery and functionalization of the QDs. In the literature, there are several reviews on the synthesis of QDs [10,32] and in this section the focus will be on aspects related to the chemistry of QDs of II/VI materials.

It was after the landmark work of Murray, Norris and Bawendi that high quality QDs became readily available in reasonable amounts [33]. These authors showed that $\mathrm{CdE}(\mathrm{E}=\mathrm{S}, \mathrm{Se} \mathrm{Te})$ semiconductor nanocrystallites, with a close control of their size, could be obtained by the injection of dimethylcadmium and the respective chalcogenide source into a hot solvent such as tri-noctylphosphine oxide (TOPO). The injection of these precursors into the hot solvent (typically between $200-300^{\circ} \mathrm{C}$ ) results in a short burst of nuclei which are generated in homogeneous solution. This rapid nucleation depletes in a large extent the reactants and limits the occurrence of further nucleations and subsequent growth occurs almost exclusively through Ostwald ripening. The molecules of the high boiling point solvents normally used also have the capability to coordinate to the nanocrystals surfaces, hence acting as barriers against the nanoparticles coalescence into bulk powders. The QDs produced by this method and similar strategies are of high quality, i.e. they consist of nanocrystals with narrow size distributions and organically capped surfaces. The size of the nanocrystals can be controlled by adjusting experimental parameters such as the type of solvent, reaction temperature and time, with the size of the particles increasing with increasing reaction temperature. Also control on the injection rate and temperature on further additions of molecular precursors to the reacting mixture, allows control of the shape and type of the polymorph observed in the final QDs[34].

The original TOPO method has been successfully implemented to coat the QDs core with a higher bandgap semiconductor (typically, ZnS). This can be achieved by injection of solutions of dimethyl or 
diethylzinc and hexamethyldisilathiane $\left((\mathrm{TMS})_{2} \mathrm{~S}\right)$ into the hot solvent containing the core $\mathrm{CdE}$ nanocrystallites [26].

The major drawback of the above method is the high toxicity and difficult manipulation associated to some of the starting chemicals. For instance $\mathrm{Cd}\left(\mathrm{CH}_{3}\right)_{2}$ is extremely toxic and pyrophoric, and these limitations become more relevant for high temperature synthesis. Although maintaining some of the conceptual characteristics of the original synthetic method [31], alternatives to this approach have emerged in the following years. One of these approaches was introduced by Trindade and O'Brien and involves the thermal decomposition of single-molecule precursors, i.e. a source compound that contains in the same molecule the elements required for the formation of the semiconductor, such as alkyldiseleno- or alkyldithiocarbamato metal complexes $[35,36]$. This method is particularly attractive when readily available air-stable precursors can be employed in a one-step process to obtain nanocrystalline materials; this has been clearly the case of the synthesis of several metal sulphides from dithiocarbamato or xantate complexes [36, 37]. This approach has also been investigated to coat CdSe QDs with ZnS and CdS, either by the thermal decomposition of the respective metal dithiocarbamate complexes [38] or via sonochemical decomposition of metal xanthates [39].

An important advance on the synthesis of QDs using TOPO related methods was carried out by Peng and Peng during their mechanistic studies of such type of reactions to produce semiconductor nanocrystals [40]. They observed that by introducing a strong ligand in the reacting mixture, such as hexylphosphonic acid (HPA) or tetradecylphosphonic acid (TDPA), $\mathrm{Cd}\left(\mathrm{CH}_{3}\right)_{2}$ was immediately converted into Cd(II) HPA/TDPA complexes which in turn could act as the cadmium source. This observation was of great relevance because these cadmium complexes can be easily obtained by dissolving other $\mathrm{Cd}(\mathrm{II})$ compounds, such as $\mathrm{CdO}$ or $\mathrm{Cd}\left(\mathrm{CH}_{3} \mathrm{CH}_{2} \mathrm{O}_{2}\right)_{2}$, in a range of organic solvents (such as a phosphonic acids, fatty acids, or amines). These cadmium compounds are easier to handle and less expensive when compared to $\mathrm{Cd}\left(\mathrm{CH}_{3}\right)_{2}$, thus facilitating the scale-up synthesis of QDs of II/VI materials using diverse types of solvents (e.g. long chain carboxylic and phosphonic acids as well as amines) [41].

\subsection{Surface modification and functionalization}

At present, the lyothermal syntheses using high boiling point solvents, are the most commonly used routes to QDs of II/VI materials. Although organically capped QDs produced by these approaches are of high quality there has been interest in developing water based synthesis of QDs with comparable quality. In fact, for a number of analytical applications, in particular biological related, stabilization of the QDs in aqueous medium is crucial. Quantum dots of mercury telluride ( $\mathrm{HgTe})$ and cadmium telluride (CdTe) with superior optical properties have been reported as interesting examples [42]. Also, aqueous based preparations of thiol-capped CdS [43], CdSe [44] and CdTe [45-47] have been reported. These generally involve the reaction between a water soluble Cd(II) salt and a chalcogenide source in the presence of thiolates which act as stabilizers similarly to the TOPO molecules in the above methods. However, in this case the capping molecules are amphiphilic molecules containing a thiol group strongly coordinated to the QDs surfaces and a polar group $\left(-\mathrm{OH},-\mathrm{COOH}\right.$ and $\left.-\mathrm{NH}_{2}\right)$, that confers compatibility with aqueous solutions to the QDs. In these methods, the availability of sources for the chalcogenide $(\mathrm{E}=\mathrm{S}, \mathrm{Se}$ and $\mathrm{Te})$ is limited and the corresponding acids $\left(\mathrm{H}_{2} \mathrm{E}\right)$, either directly or 
during its preparation ( $\mathrm{NaHE}$ ), have been used. Although the lack of source alternatives have hampered fast developments of these aqueous-based methodologies, they are promising synthetic strategies to expand the range of water soluble QDs. For instance, precursors like $\left(\mathrm{NH}_{4}\right)_{2} \mathrm{Te}$ (obtained from reaction between Te metal and ammonium hydroxide in the presence of aluminium) have been introduced recently for the synthesis of CdTe [48].

Another possibility to obtain water compatible QDs is to promote appropriate chemical reactions at the surface of organically capped QDs. Phase transfer of such QDs to aqueous solution requires surface functionalization with hydrophilic ligands. To this end, two main general routes have been developed: i) exchange of the native hydrophobic ligands by hydrophilic molecules, commonly designated as 'cap exchange', or ii) encapsulation of QDs in a heterofunctional coating, through hydrophobic interaction with the capping molecules.

In the first approach, the 'cap exchange' involves the replacement of native capping molecules (e.g. TOPO) with ambidentate organic ligands. These are bifunctional molecules that on one hand can coordinate to the QDs surface trough a soft acidic group (usually a thiol) and on the other hand have hydrophilic groups (for example carboxylic or aminic groups) which point outwards from the QDs surfaces to bulk water molecules. Regarding this matter, several monothiolated ligands have been used but decrease of luminescence quantum yields overtime has been reported [49-53]. A possible explanation relates to the coordination of water molecules to the QDs surface. In fact substitution of monothiols by polythiols or phosphines usually improves stability [54, 55]. Improved stability can also be achieved by "cap exchange" and encapsulation in dendron boxes [56,57]. These supramolecular structures offer superior protection to the QDs due to the cross-linking of the dendron ligands.

The second strategy that can be used to promote hydrophilicity of capped QDs involves the growth of amorphous silica shells which can be further functionalized with other molecules or polymers [58]. Silica coating starts with a 'cap exchange' reaction where the native ligands are substituted by silanes, e.g. mercaptopropyltris(methyloxy)silane (MPS). Condensation of the methoxysilane groups to the dots surfaces occurs through hydrolysis reaction in alkaline media. Further growth of the $\mathrm{SiO}_{2}$ shells can be achieved through hydrolysis of a silicon alkoxide (e.g. TEOS) using the Stöber method. The reactivity of amorphous $\mathrm{SiO}_{2}$ has wide use in different branches of chemistry and this siloxane chemistry can be employed to anchor a variety of organic molecules to the $\mathrm{SiO}_{2}$ surfaces [59].

The exchange of the native capping ligands is not a necessary condition to tailor hydrophilic surfaces in QDs. Encapsulation of the QDs is also possible by 'wrapping' the dots with amphiphilic macromolecules. The hydrophobic moieties of the cap interact with the native TOPO molecules (or analogous functional ligands) at the QDs surface, whereas the hydrophilic outer block points to the aqueous phase. This type of QDs encapsulation has been reported for amphiphilic copolymers [60-62], phospholipids [63-65] and 'bulky' molecules such as tetrahexyl ether derivatives of $p$ sulfonatocalix[4] arene [66, 67]. However, this method is not restricted to amphiphilic molecules. For instance, QDs can be encapsulated with polymers via phase separation in oil-in-water microemulsions in the presence of a surfactant [68]. Solvent evaporation and crosslinking of the polymer structure results in robust nanostructures of polymer encapsulated QDs [68].

The combined use of polymers and QDs can produce an endless number of nanocomposites with diverse properties. Additionally to the QDs' unique properties, there is interest in exploiting synergistic effects which might arise from their intimate combination with the polymer matrix. For 
example, the colloidal stability of organically capped QDs in organic media, provided by the native capping molecules, can be exploited to prepare polymer nanocomposites by in-situ miniemulsion polymerization in the presence of the dots. This technique was prototyped with a series of inorganic nanofillers, such as $\mathrm{TiO}_{2}$ [69-71], $\mathrm{SiO}_{2}$ [72] and carbon black powders [73], in which the nanofillers have been dispersed in the monomer without any previous surface treatment. More recently, the encapsulation of organically capped nanoparticles (QDs and nanometals) by in situ miniemulsion polymerization was demonstrated for PS [74, 75], PBA [74, 76] and PS/PMMA [77]. The optical behavior of the nanocomposites seems to depend on a number of parameters which include the type of polymer used. Studies on the luminescence behavior of homogeneous CdSe/PBA show highly luminescent nanocomposites unlike their polystyrene analogues [78].

Note that in this strategy there is no formation of strong covalent bonds between the capping molecules and the polymer molecules. In fact, the experimental conditions need to be optimized in order to limit migration of the dots to the surface of the polymer beads. Nevertheless, these nanocomposites contain functional groups that allow surface modification by relatively simple methods. For example, PBA based nanocomposites contain polyester groups that can be hydrolyzed to provide carboxylic functionalities at the surface and hence, allowing biofunctionalization with antibodies via formation of peptide linkages [76].

The functionalization of QDs with polimerizable groups can induce graft copolymerization with vinyl monomers, thus leading to a homogeneous distribution of QDs inside polymer latexes. The introduction of vinyl-functionalized $\mathrm{CdSe} / \mathrm{ZnS}$ in styrene droplets, by exchange of the native capping by 4-mercaptovinylbenzene, has been investigated in miniemulsion polymerization but still migration of the dots to the surface was observed along with the deterioration of the photoluminescence of the final material [75]. An hybrid method that comprises in a first step the silica encapsulation of the $\mathrm{CdSe} / \mathrm{ZnS}$ dots, followed by functionalization of the silica at the surface with methacryloxypropyltrimethoxysilane (MAS) and then graft copolymerization, gave core-shell QDspolymer nanocomposites, but again there was a decrease on the luminescence of the nanomaterials [79].

Figure 3 summarizes some of the innovative chemical strategies that have been used to produce organically capped and/or polymer encapsulated QDs, as discussed above. However, there is still a number of practical issues which need improvement. The broad particle size distribution of the nanocomposites and difficulties to obtain an homogeneous distribution of QDs inside the polymer particles, sometimes associated to a detrimental effect on the optical behavior of the nanocomposites, led researchers to introduce new polymerization methods, such as surface initiated controlled/living polymerization. This type of approach has been successfully demonstrated for the controlled growth of dense polymer brushes from several inorganic surfaces, such as gold, [80, 81] $\mathrm{SiO}_{2},[82,83]$ and magnetic nanoparticles (magnetite/PMMA) [84]. More recently, these surface initiated methods have been investigated to attain the controlled growth of polymeric phases from the surface of functionalized QDs. As such, there is intense research in using synthetic techniques, such as additionfragmentation chain transfer polymerization (RAFT) [85], nitroxide-mediated polymerization (NMP) [86] and atom-transfer radical polymerization (ATRP) [87], applied to the functionalization of QDs surfaces. 


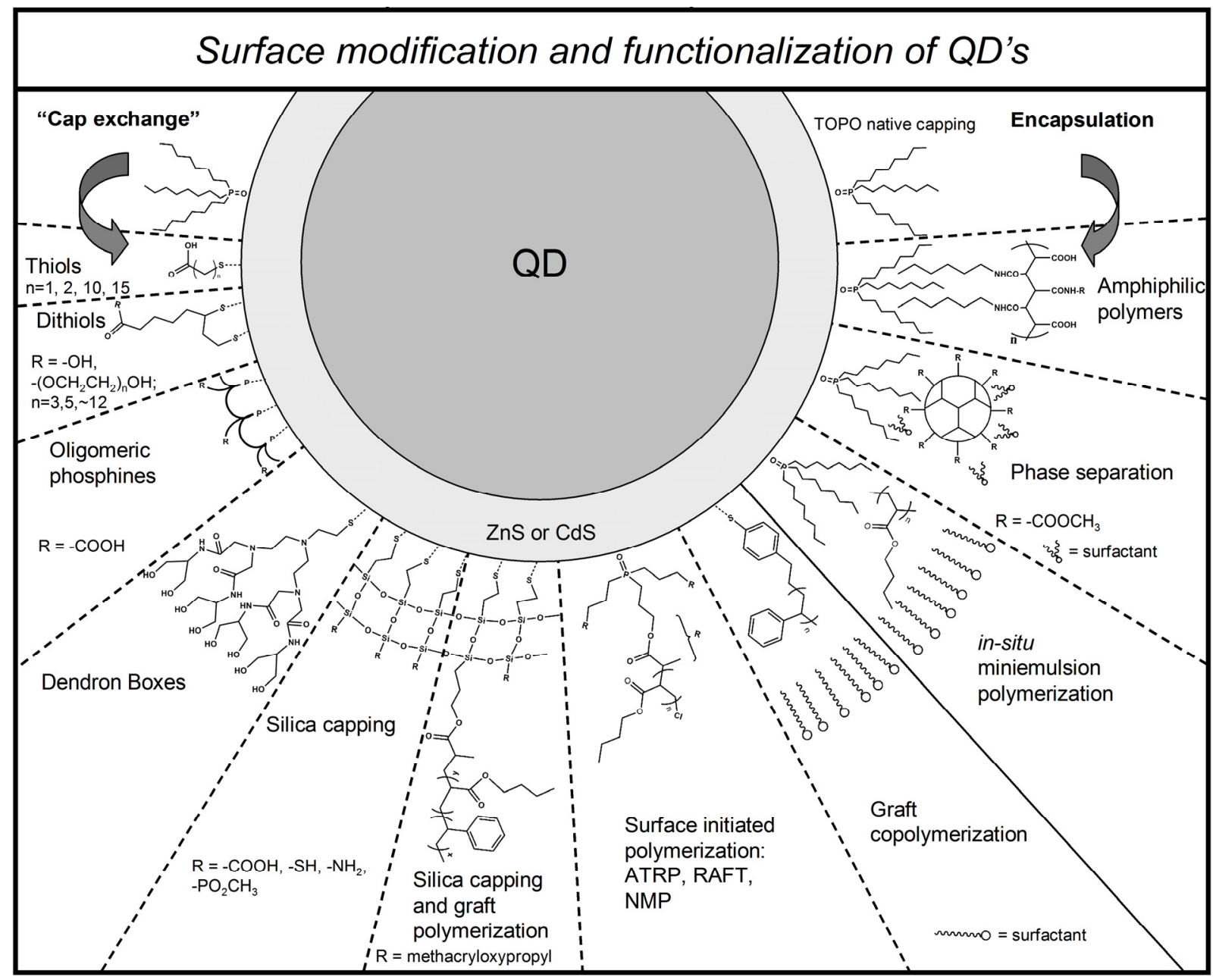

Figure 3. Scheme illustrating some of the methods for chemical surface modification of QDs.

\section{Sensing mechanisms and applications}

The combination of appealing optical properties with the facility of bioconjugation make semiconductor nanocrystals an attractive tool for a wide variety of applications. In the field of optical sensors, the ability to tune the QDs optical properties and to tailor the chemical and biological characteristics of their surface contribute to an increasing number of sensing strategies. An overview of the use of QDs in different fields of optical sensing will be addressed in the following sections. Focus will be given to key developments and latest reported advances, particularly those with potential for future optical fiber sensing applications.

\subsection{Physical sensors}

While at present, some of the most attractive features of QDs are being explored in biosensing applications, their temperature behavior renders them excellent temperature probes with many potential uses [88-90].

Walker et al. first characterized the temperature response of colloidal quantum dots immobilized in polymer hosts [91]. In this work, core-shell CdSe/ZnS nanocrystals entrapped in poly(lauryl methacrylate) - PLMA, were subjected to temperature changes while excited with a $488 \mathrm{~nm}$ laser line. 
Monitoring the photoluminescence of the doped polymer membrane revealed that the peak wavelength $\left(\lambda_{\text {peak }}\right)$, the linewidth, and the intensity of this emission were all strongly temperature dependent. A blue shift of $20 \mathrm{~nm}$ in the peak emission was observed, while the temperature decreased from $42^{\circ} \mathrm{C}$ to $-173^{\circ} \mathrm{C}$. In the same temperature range, the FWHM decreased from $26 \mathrm{~nm}$ at higher temperatures to $22 \mathrm{~nm}$ at lower temperatures. However, the strongest effect was observed in the photoluminescence intensity which increased by a factor of five as temperature decreased. In particular, for the near ambient temperature range $\left(5^{\circ} \mathrm{C}\right.$ to $\left.40^{\circ} \mathrm{C}\right)$, the photoluminescence intensity change was linear and in the order of $-1.3 \%$ per ${ }^{\circ} \mathrm{C}$. It was shown that, in this temperature range, the wavelength shift was small $(\sim 2 \mathrm{~nm})$ and the FWHM variation was negligible $(<1 \mathrm{~nm})$. All the changes were reversible with temperature. Good reproducibility was observed even after 3 hours of continuous irradiation, demonstrating high photostability of immobilized CdSe/ZnS QDs. This temperature behavior was essentially identical for QDs excited at different wavelengths (460, 530, and $580 \mathrm{~nm}$ ) and in different host materials (polymer and sol-gel).

This work established the suitability of QDs as temperature references in luminescence based sensing applications. The fact that sensing was achieved within a solid host opened a pathway for future works with optical fiber and waveguide platforms.

\subsection{Chemical sensors}

In contrast with the fast widespread use of QDs in bio-imaging and sensing applications, the use of such semiconductor nanoparticles as chemical sensing probes had a somewhat slower start [11]. Until very recently, few reports had been published concerning the application of QDs as luminescent indicators for detection of chemical species. Nevertheless, the luminescence of core QDs can be very sensitive to the surrounding chemical environment. This can be the path for using nanocrystals as chemical sensors, provided that some selectivity can be achieved. The desired selectivity can be controlled by chemically tailoring the outer surface of the semiconductor nanoparticles. The methods referred to above to control the nanocrystals solubility and stability are the starting point from which further functionalization will allow QD-based sensing indicators. Presently, a significant number of chemical sensing strategies using QDs is being explored [14].

Coating the QDs' surfaces with suitable ligands can have a strong effect on its luminescent response to specific chemical species. In fact, the presence of the analyte can quench or enhance the nanocrystal luminescence, depending on the functionalization strategy. In a representative work, Chen and Rosenzweig [92] were able to alter the selectivity of CdS nanoparticles to respond either to $\mathrm{Zn}^{2+}$ or $\mathrm{Cu}^{2+}$ ions, simply by changing their capping layer. They showed that, while polyphosphate-capped $\mathrm{CdS}$ quantum dots responded to almost all mono and divalent metal cations (showing no ion selectivity), thioglycerol-capped $\mathrm{CdS}$ nanocrystals were sensitive only to $\mathrm{Cu}^{2+}$ and $\mathrm{Fe}^{3+}$. The QDs luminescence was quenched by iron(III) and copper(II). However, it was not affected by other ions occurring at similar concentrations. On the other hand, the luminescence emission of L-cysteinecapped CdS quantum dots was enhanced in the presence of zinc ions but was not affected by other cations such as $\mathrm{Cu}^{2+}, \mathrm{Ca}^{2+}$ and $\mathrm{Mg}^{2+}$. Different mechanisms were responsible for the luminescence quenching or enhancement, ranging from electron-transfer to filter effects. The different processes, depend on the QD-probe/ion combination and are discussed in detail in [92]. Quenching by iron(III) 
was observed, which interfered with the detection of copper and zinc, however, this was attributed to an inner filter effect, and could be eliminated by adding fluoride ions to the solutions in order to form a colorless complex. Using this set of QD probes, the authors established the selective detection of zinc and copper in physiological buffer samples, where several other metal ions were present. Quantitative measurements were performed where detection limits of $0.8 \mu \mathrm{M}$ and $0.1 \mu \mathrm{M}$ were achieved for zinc(II) and copper(II), respectively. This was claimed to be the first use of semiconductor nanoparticles as selective ion probes in aqueous samples. More recently, the detection of copper in physiological buffer solutions, with a detection limit of $0.1 \mathrm{nM}$, was achieved by using $\mathrm{CdSe} / \mathrm{ZnS}$ nanocrystals modified with bovine serum albumin [93].

The same principle was applied for the detection of inorganic anions. The use of surface modified $\mathrm{CdSe}$ quantum dots functionalized with tert-butyl- $N$-(2-mercaptoethyl)-carbamate (BMC) groups for the determination of cyanide was demonstrated by W.J. Jin et al.[94]. Surface modification can also promote sensitivity to other ionic species. The detection of a diversity of ions like Fe(III), $\operatorname{Ag}(\mathrm{I})$, $\mathrm{Mn}(\mathrm{II}), \mathrm{Ni}(\mathrm{II})$ or I-, by means of a variety of processes such as inner filter effects, non-radiative recombination pathways, electron-transfer processes and ion-binding interactions, has been reported by several authors and was adequately reviewed [14].

More complex chemical species have also been determined using different cappings at the QDs surfaces. In a recent work, G. H. Shi et al. demonstrated that QDs coated with oleic acid were readily quenched by a diversity of nitroaromatic explosive molecules, such as 2,4,6-trinitrotoluene (TNT) or nitrobenzene (NB) [95]. Different quenching behaviors were observed for different molecules. Nevertheless, in most cases, modified Stern-Volmer equations could be used to provide linear calibration curves. Time domain measurements showed that static quenching was the dominant process since no change in luminescence lifetime was observed in the presence of the analyte. Detection limits of $10^{-6}$ to $10^{-7} \mathrm{M}$ seem poor when compared to previously reported methods [96]. Nevertheless the detection mechanism is comparatively simpler and has room for improvement.

The sensing of gases using polymer embedded nanocrystals has also been demonstrated [97]. The authors found that photoexcitation of the electrons in QDs could make the ligands monolayer readily permeable to gas molecules. Under photoirradiation, the PL properties of the nanocrystals responded to the environment in a reversible, rapid, and species-specific fashion. The photo-stimulated response was thought to be related to photon-phonon coupling of the optical absorption and emission processes occurring in the nanocrystals.

Based on the same principle, more recently Potyrailo and co-workers showed that when different sized TOPO capped CdSe QDs were incorporated into a polymer film and photoactivated, CdSe nanocrystals of different sizes unexpectedly demonstrated distinct photoluminescence response patterns when expososed to polar and non-polar vapors in air [98]. The authors suggested that by using principal component analyses (PCA) of the spectral response of a multi QD doped film, a selective gas sensor could be obtained, thus introducing the possibility of multiparameter gas sensing. The principle was demonstrated by submitting a PMMA film doped with CdSe QDs with distinct average sizes (2.8 $\mathrm{nm}$ and $5.6 \mathrm{~nm}$ diameter), to different concentrations of methanol and toluene. In order to obtain selective chemical response from individual response patterns of the two distinct QD populations, principal components analysis technique was applied to different wavelength ranges. Plots obtained demonstrated that responses of the sensor film to the different vapors were well separated in the PCA 
space, allowing for easy discrimination. Such work has the potential to complement existing solvatochromic organic dye sensors with more photostable and reliable sensor materials.

Uncoated QDs are sensitive to the environment $\mathrm{pH}$. Nonetheless, they are also prone to oxidation and photodegradation. While the protective overcoating renders the nanocrystals highly photostable, their $\mathrm{pH}$ sensitivity is very much reduced. However, functionalization of the dots surface with $\mathrm{pH}$ sensitive ligands can render the resulting nanoparticles responsive to acidity levels. The functionalization of CdSe/ZnS QDs with a chromophore whose absorption spectrum shifted according to the surrounding $\mathrm{pH}$ has been reported [99]. The absorption shift changed the relative overlap with the QDs' emission modulating the fluorescence resonance energy transfer (FRET) efficiency according to $\mathrm{pH}$. Using this method the authors demonstrated an approximately linear variation of the QDs' luminescence intensity within the $\mathrm{pH}$ range 3-11 (a 30\% intensity change was observed in the full range). It was suggested that similar organic ligands could be designed to alter their absorption and redox properties in response to target analytes other than $\mathrm{H}^{+}$(or $\mathrm{OH}^{-}$), changing the luminescence of their conjugated QDs. A potential problem of this strategy lies in the fact that purely intensity based measurements are prone to be affected by several sources of optical power drift. Although the QDs' luminescence lifetime was shown to be $\mathrm{pH}$ dependent, average values in the order of 15 ns were reported. Therefore the implementation of intensity independent lifetime or frequency domain interrogation techniques would require very fast optoelectronics.

In a more sophisticated approach, a QD based ratiometric probe was designed by Bawendi's team [100]. This was achieved by conjugating $\mathrm{CdSe} / \mathrm{ZnS}$ overcoated with TOPO with a NIR luminescent squaraine dye. $\mathrm{pH}$ modulated FRET resulted in a luminescent emission displaying an isobestic point at $640 \mathrm{~nm}$ enabling, therefore, the implementation of ratiometric detection schemes. The generalization of this approach to sensing different biochemical species is possible as the narrow, size-tunable emission spectrum of QDs, acting as donors, can be matched with the acceptor absorption features of an analyte sensitive dye, maximizing FRET efficiency.

Overall, the reported different approaches indicate the feasibility of using surface modified QDs as analytical probes for the determination of biochemical species. Nevertheless, most experiments took place in an aqueous media while fiber sensing instrumentation most often requires the sensing dye immobilization.

Very recently, however, Ruedas-Rama et al. have reported the development of multi-ion sensing using different combinations of QDs with selected ionophores or organic fluorophores embedded in a polymeric composite material [101]. In this work, the authors explored the differences in efficiency of fluorescence resonance energy transfer between quantum dots and proximal organic dyes in order to discriminate ion sensitive emission signals, while using a single excitation wavelength. In particular, by co-immobilizing in acrylic nanospheres green emitting QDs with lucigenin, or valinomycin and a selected chromoionophore, $\mathrm{Cl}_{2}$ sensitive and $\mathrm{K}^{+}$selective sensors could be created, respectively. Embedding the resulting nanospheres in a common polymeric matrix, dual sensitive ionic sensors with no cross talk could be created. In the presence of $\mathrm{K}^{+}$and $\mathrm{Cl}_{2}$ the fluorescence of lucigenin is quenched, and QDs act as donors interacting with the deprotonated chromoionophore (acceptor) by FRET. Because each ion was sensed by a different independent mechanism, the resulting luminescent response of the ensemble allowed the authors to independently monitor the presence of each one of the analytes in different spectral regions (see Figure 4). 

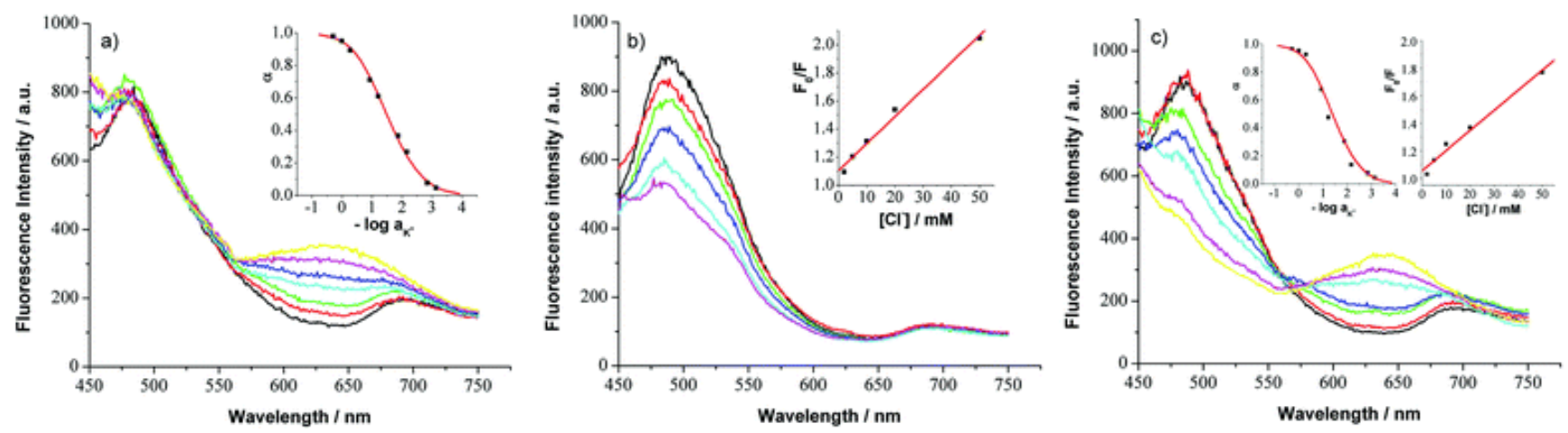

Figure 4. Emission spectra of acrylic/QDs nanospheres acting as: (a) single $\mathrm{K}^{+}$sensors after addition of $\mathrm{KHCO}_{3}$ solutions. (b) Single $\mathrm{Cl}^{-}$sensors after addition of $\mathrm{NaCl}$ solutions. (c) $\mathrm{K}^{+} / \mathrm{Cl}_{2}$ multi-ion sensors after addition of $\mathrm{KCl}$ solutions. Concentrations: $0 \mathrm{mM}$ (black), $2 \mathrm{mM}$ (red), 5mM(green), 10mM

(blue), $20 \mathrm{mM}$ (cyan), $50 \mathrm{mM}$ (pink) and $200 \mathrm{mM}$ (yellow). Fluorescent signals were recorded between 450 and $750 \mathrm{~nm}\left(\lambda_{\text {ex }}=400 \mathrm{~nm}\right)$ with a spectrofluorimeter (Cary Eclipse, Varian) in a 96-well microplate. For all samples the $\mathrm{pH}$ was fixed at 7.0 by using phosphate buffer. [101] - Reproduced by permission of The Royal Society of Chemistry.

A similar strategy was used in order to develop a selective $\mathrm{Na}^{+}$sensor. This was achieved by codoping polymer microspheres with TOPO capped CdTe QDs, a sodium ionophore X, a Nile Blue derivative ETH 2439 and a lipophilic tetraphenylborate cation exchanger. These probes were shown to respond to $\mathrm{Na}^{+}$in the $10^{-4}$ to $0.1 \mathrm{M}$ range, at $\mathrm{pH} 4.8$ with excellent selectivity towards common interferences [102]. The microspheres were prepared by sonic particle casting, a method that was shown to enable the implementation of dual doped particles having precisely controlled amounts of two types of nanocrystals.

The fabrication of composite materials such as these, is a very versatile technology that can be explored for simultaneous sensing of a diversity of chemical species, both in solution based assays or in solid platforms.

In a different approach, molecular imprinting technology was used to render the photoluminescence of semiconductor nanocrystals sensitive to specific molecules [103]. If the synthesis of a polymer is made in the presence of an imprint or template molecule, cavities will be produced in the polymer, which are highly selective for the imprint. C.I. Lin et al. prepared molecular imprinted photoluminescent polymers (MIP) using CdSe nanoparticles functionalized with 4-vinylpyridine. Several polymers containing the QDs were imprinted with different template molecules (caffeine, uric acid, L-cysteine). The resulting solid polymers were ground to a fine powder and sieved. The template molecules were then extracted from the obtained powder.

The detection of the analytes was performed by incubation of the MIPs with the corresponding template molecules in aqueous solutions. It was observed that the photoluminescence emission of the MIPs was strongly quenched in the presence of the corresponding templates. However, no quenching occurred in the presence of other molecules. Strong quenching was observed for the caffeine imprinted polymer in the presence of caffeine while the presence of similar molecular structures like theophylline and theobromine had no effect on the photoluminescence. Also, a control polymer, with no imprint, 
showed no change in the QDs photoluminescence. These results demonstrated that it is possible to couple nanocrystals with the selective recognition capability of MIPs, opening several possibilities for the application of semiconductor nanoparticles in optical chemical sensing.

The fact that these sensing principles were demonstrated in solid hosts, where the sensing indicators were hydrophobically, or covalently retained, introduces the possibility of transposing these technologies to optical fiber or planar waveguide platforms.

\subsection{Biosensors}

The unique optical properties of QDs established them as appealing alternatives to traditional organic dyes in the context of biotechnology, offering potentially greater performance in fluorescence based immunoassays and bio-imaging applications $[12,13,104]$. The introduction of QD technology in the biological domain involves its chemical stabilization, the control of its hydrophobic/hydrophilic properties and, finally, its conjugation with a biomolecule of interest, which will define its biofunctionality. All these processes will influence the luminescence properties of the original QDs. Nevertheless, several successful strategies have been developed with covalently (or non-covalently) bound biomolecules to the surfaces of QDs, and some of these bioconjugated QDs are already commercially available $[105,106]$. The size of each QD, which is much larger than that of a single dye molecule, is compatible with the simultaneous conjugation with more than one biomolecule. This provides QDs with the potential for an increased sensitivity, multi-analyte detection with single QD species, and other new functionalities. On the other hand, the increased size also brings some concern about interference with the biomolecules mobility and functionality [12].

In one of the first reported bio-assay applications of CdSe/ZnS QDs, these were covalently coupled to a protein and exhibited 20 times more luminescence intensity when compared to Rhodamine [107]. Additionally, the QDs were reported to be 100 times more resistant to photobleaching. This allowed the authors to perform ultrasensitive detection at the single-dot level. However, an on/off behavior of single dot emission was observed. This fluorescence 'blinking', also observable in some dye molecules, can have off-periods of several seconds in QDs which can compromise single dot measurements. Nevertheless, when QD ensembles are observed, this effect goes unnoticed due to averaging of their luminescence emission [108]. While preserving the optical properties of the QDs, the authors also demonstrated that the attached biomolecules were still active and able to recognize specific analytes. The first example of a nanocrystal in-vitro immuno-assay was the case of quantum dots labeled with IgG antibodies, which were recognized and agglutinated by polyclonal anti-igG. The authors also demonstrated cell labeling by transporting QD-transferrin bioconjugates into cultured HeLa cells via receptor-mediated endocytosis.

The potential of QDs for multicolor assays was first demonstrated by Bruchez et. al. when two different sized $\mathrm{CdSe} / \mathrm{CdS}$ QDs, emitting green and red luminescence, were specifically bound to different parts of 3T3 mouse fibroblast cells, and were excited by a single optical source [7]. In this pioneer experiment, some nonspecific binding was observed. Higher degrees of specificity are required in in-vivo applications where background biomolecules can generate false positives. Stabilization and conjugation techniques rapidly evolved and higher levels of specificity have been achieved by different authors [60, 63, 109]. 
In a pioneer work, Mattoussi and coworkers [109] prepared CdSe/ZnS QDs with mixed protein adaptors to provide nanocrystals with the specific recognition capability of antibodies. A representative example of such labeling/sensing platform is illustrated in Figure 5. Positively charged avidin and maltose-binding protein, with a positively charged tail (MBPzb), self assembled on the negatively charged surface of QDs capped with dihydrolipoic acid (DHLA), was bind to biotinylated antibodies specific for Pgp. These QD probes were selective to cells that expressed detectable levels of labelled PgP-GFP (green fluorescent protein). The approach is a generic strategy since different QDs can be conjugated to a wide range of antibodies, thus providing a whole new set of highly specific probes capable of very specific labeling target molecules in imaging and sensing applications.

Since this seminal work, many similar strategies have been reported for highly specific in vivo applications. Xiaohu Gao et al. have developed multifunctional QD probes rendering them into efficient cancer markers in living organisms [62]. CdSe/ZnS protected by TOPO ligands were first encapsulated with an amphiphilic triblock copolymer for further protection against enzymatic degradation, thus avoiding particle aggregation and detrimental luminescence effects as well. Different labeling solutions were then explored by coating the resulting nanoparticles with polyethylene glycol (PEG) and a variety of tumor-targeting ligands, such as peptides, antibodies or small molecule inhibitors. The developed luminescent tags were shown to be extremely photostable preserving their luminescence characteristics even in a $\mathrm{pH}$ range from 1 to 14 or in different salt conditions $(0.01 \mathrm{M}$ to $1 \mathrm{M})$. In vivo tumor labeling was then demonstrated in live mice where the QD probes were delivered to tumors by both passive and active targeting mechanisms exploring the enhanced permeability and retention effect of cancer cells, or the specificity of antibodies (in particular QDs tags were conjugated with a prostate-specific membrane antigen -PSMA-). Because many copies of the same labelling ligand can be linked to single dots, binding affinities could be increased by ten orders of magnitude as compared to single ligand markers. The authors also presented detailed studies on the in vivo behavior of the developed QD probes, including their biodistribution, nonspecific uptake, cellular toxicity and pharmacokinetics. The simultaneous imaging of multicolor QD-encoded microbeads in living animals was also demonstrated and compared with green fluorescent protein (GFP). QDs showed a much higher performance offering high contrast against tissue autofluorescence and long term imaging capabilities.

Many other possibilities have been reported by other authors, from QD stained polymer beads for multiple color-code cell labeling, to the use of high contrast non-specific vascular imaging and the clear imaging and delineation of sentinel nodes using type II QDs (emission 700-800 nm) (this enables the possibility of providing in situ visual aid for surgical removal of small lesion otherwise unnoticeable)[110-112]. Presently, QDs based probes are already established tools in many medical imaging applications. However, concerns have been raised over the nanocrystals' toxicity that may hinder their clinical use. Although cadmium ions alone can be highly toxic to cells, coating the coreshell dots with polymer and lipid layers minimizes the risk of contamination. However, while adequately coated QDs showed little or no toxicity in a variety of a cells and living animals, when exposed to UV excitation for long periods of time, they can become toxic to cells [113]. UV light seems to induce semiconductor degradation by photolysis leading to the release of highly toxic cadmium ions. Because the unique features of QDs are decidedly attractive for clinical use, lately a growing number studies regarding toxic effect of semiconductor nanocrystals and ways to circumvent 
them, such as the use of QDs without heavy metals or application of lower energy excitation, have been reported [114, 115]. Nevertheless, in a near future, research on the clinical use of QDs will be certainly directed for in vitro applications, although toxicity concerns should also be taken into account.

(a)
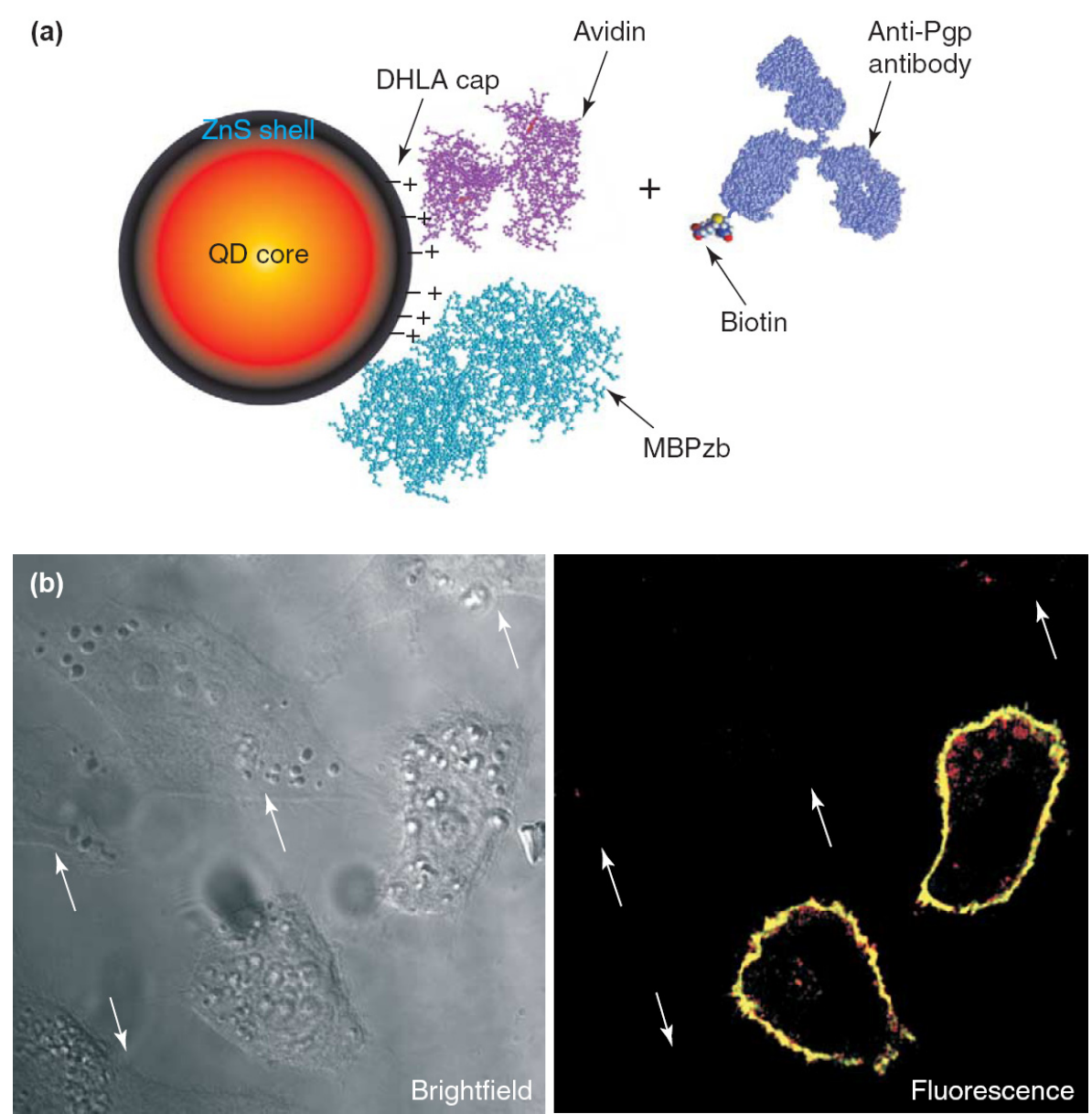

Figure 5. Specific labelling of live cells with QDs. (a) Schematic representation of the QD antibody conjugation strategy. (b) Labelling of cell membranes with the QD bioconjugates: only cells expressing detectable levels of Pgp-GFP were labelled, those that did not express Pgp-GFP (marked with arrows) did not bind with QD probes. Yellow coloring in the fluorescence image indicates an overlap of green (Pgp-GFP) and red (QDs bioconjugate) fluorescence emission. (See Ref. [109, 116] for further details) Reproduced from Trends in Cell Biology, 14, Jaiswal, J. K. and Simon, S. M., Potentials and pitfalls of fluorescent quantum dots for biological imaging, 497-504, Copyright (2004), with permission from Elsevier.

The strategies developed for specific labeling in imaging applications were quickly adapted to the improvement of biosensors. The CdSe/ZnS QD-antibody conjugates developed by Matoussi et al. [8] were used in several direct, sandwich and competition fluorescence based immunoassays, in order to detect different toxins (staphylococcal enteroxin B, cholera toxin) and small molecule explosives like TNT. In the several assays performed, limits of detection were achieved that were, at least, as low as the ones obtained with dye-based assays. The same authors performed the first study about fluorescence resonance energy transfer in QD-protein conjugates. Nanocrystals were used as energy 
donors to acceptor dye molecules that were attached to the conjugated proteins. This configuration enabled the exploration of the influence of parameters such as donor-acceptor spectral overlap and donor-acceptor ratio on the FRET efficiency [117]. The resultant FRET enhancement contributes for increased assay sensitivity. These studies were intended to evaluate the possibility of quantifying analyte concentrations by fluorescence quenching. In sequence of the results obtained, a prototype of a QD FRET sensor for sugar detection was presented. Each QD was conjugated, via His-Zn coordination, with 15 to 20 maltose binding proteins (MBP) and with further processing a QSY-9 quencher was bounded to each MBP. The concentration dependent quenching of the QD emission was obtained for two reasons: (1) the QSY-9 absorption overlapped perfectly with the emission of the 555 $\mathrm{nm}$ emitting QDs, and (2) its separation distance from the QD center was within the range of FRET critical radius. When maltose was added to the solution, the quencher was displaced and FRET was interrupted. An apparent binding constant of $7.0 \mu \mathrm{M}$ was found from the titration curve with maltose. A response was obtained when only certain sugars have been used, showing that the QD-MBP conjugate kept its specificity. These results confirmed QDs as excellent FRET donors, thus establishing a new tool for sensitive and specific biosensing.

These seminal studies and applications on QD based FRET mechanisms were widely explored in a diversity of configurations where the nanocrystals acted either as donors or acceptors. This later approach is not very effective because the wide absorption spectra of QDs makes it very difficult to avoid direct excitation of the QDs by the excitation source. The different schemes for biosensing using QD FRET were recently reviewed by Rebecca C. Somers et al. [17] and include nucleic acid recognition by hybridization with DNA labeled acceptors, nanocrystal to nanocrystal FRET, conjugation with analyte sensitive chromophores or luminophores, among others. Such variety of solutions makes FRET-based schemes the main mechanism for QD-based biosensing.

While early works were very generic proof of principle assays, more recently, systematic approaches to sensing important chemical or biological species have been addressed. In particular, a diversity of strategies was reported for detection of different proteins and virus species (eg. H9 avian influenza virus) $[9,118,119]$. While in most works described, sensing is made with the QDs dispersed in a solvent, recent works have reported the use of QDs doped polymer beads for immunodetection $[120,121]$. In a particular example, the surface of polymer beads was functionalized with human $\operatorname{IgG}$ and used to detect goat antihuman-IgG labled with a luminophore [121]. In this particular case, the dual QDs emission was used to univocally identify a particular group of beads, making way to multiparameter immuno-sensing.

A particular important biosensing application of QDs is the case of glucose sensing via luminescence. In the first reported QD glucose sensor, nanocrystals functionalized with carboxylic groups at their surfaces showed a decrease in fluorescence upon the introduction of a viologen quencher [122]. The author then observed a strong recovery of the luminescence intensity as increasing amounts of glucose were added to the solution. Glucose binding to the boronic acid substituted viologen quencher/receptor introduced electronic and steric changes that reduced the quenching ability. Non-linear Stern Volmer plots were obtained allowing for sensor calibration. In a different approach, MSA (D,L-mercaptosuccinic acid), caped QDs were used to probe the change in acidity resulting from the oxidation of glucose by the enzyme glucose oxidase, thus allowing for glucose quantification [123]. 
The unique features of QDs have established them as reference tools in imaging and sensing applications. Although most of the works reported refer to 'in solution' assays, some important progresses were described where biosensing took place using polymer encapsulated QDs. Such strategies have a strong application potential in waveguide based instrumentation.

\section{Optical fiber Sensing}

Optical fiber technology can introduce some interesting features in optical sensing applications. Real-time remote detection, miniaturization, immunity to electromagnetic interference and multiplexing ability are some of the most important ones. This has long been recognized by the scientific community and, presently, optical fiber sensing is already an established technology with many applications in industry, environmental monitoring and in the medical field [124, 125]. Currently this technology has already a considerable commercial market with a growing number of companies making its appearance. This is especially true for the measurement of physical parameters like strain temperature or pressure. Nevertheless, as a result of a strong research effort and many technological advances in areas like materials science, immobilization chemistry and optoelectronics, the use of optical fibers in a wide range of chemical and biological sensing applications has been enabled[126, 127]. In this context, luminescence based sensors are by far the most representative. In fact, in recent years, some examples of optical fiber analytical instruments for the determination of biochemical parameters by luminescent methods became commercially available (e.g. Oxygen and $\mathrm{pH}$ sensitive). However, in spite of great advances, some sensors suffer from limitations. Among others, leaching and photo-bleaching of the sensing dyes usually are important problems that limit long term stability and, therefore, the reliability and commercial viability of the sensing device [128, 129].

In this context, the unique features of QDs are highly attractive for guided wave sensing platforms such as integrated optics and optical fiber. In fact, their unsurpassed photostability and multiplexing capability can provide new interesting solutions for fiber sensing. In spite of this, to date, the application of QDs in optical fiber technology remains largely unexplored. Nevertheless, very recent progresses have been reported that will be addressed below.

\subsection{QDs' immobilization at solid surfaces}

A key step in luminescence based sensing applications using a solid platform, such as an optical fiber, is to immobilize the sensing indicators at the surface. In an ideal membrane, the sensing dyes should be encapsulated while retaining their sensing properties, avoiding leaching into the solution and simultaneously allowing a chemical equilibrium to be established with the probed media. This is a challenging task and very often some compromises need to be considered. In this context, the immobilization of colloidal QDs in solid hosts is a necessary step towards the development of guided wave QD sensing devices.

Sol-gel methods are extremely versatile because they enable the encapsulation of sensing dyes in a porous matrix by addition of the dyes prior to the polymerization stage. Choosing the right precursors allows to obtain materials with different properties. In particular, by using diverse Si alkoxides, porous $\mathrm{SiO}_{2}$ glasses membranes can be obtained that are highly compatible with guided wave devices. 
Different sol-gel procedures have been proposed to dope materials with QDs ([14] and references therein). However, achieving immobilization while maintaining the QDs unique optical properties is not an easy task. In early attempts the sensitivity of QDs to the surrounding environment often led to broad size distributions and poor stability [130]. Litran et al. have reported the preparation of $\mathrm{CdS} / \mathrm{SiO}_{2}$ xerogel composites using a sonocatalytic method. $\mathrm{Cd}(\mathrm{II})$ doped monoliths were first prepared by ultrasound-promoted hydrolysis and were subsequently exposed to an $\mathrm{H}_{2} \mathrm{~S}$ atmosphere, at high temperature in order to induce the formation of $\mathrm{CdS}$ nanocrystals within the solid host. The authors showed that this technique led to matrices with finer porosity allowing to obtain narrower QDs size distribution. In addition, thermal annealing at high temperature allowed to obtain long term stability of the optical properties. Nevertheless, at high concentration the aggregation of semiconductor particles lead to loss of quantum confinement effects and consequent spectral broadening [131, 132]. In addition, the high temperature that is necessary for simultaneous synthesis and immobilization led to the formation of dense glasses. While these glasses can find application in optoelectronic devices or solar cell implementation, its use in most sensor applications is precluded. Several approaches have been investigated aiming to overcome these limitations. Recent progresses include the use of supercritical $\mathrm{CO}_{2}$ drying allowing to obtain highly porous aerogel structures amenable to further functionalization and liquid phase interactions [133].

An efficient process to obtain concentrated and monodispersed quantum dots in a solid host is to prepare QDs in a previous step and then adding them to the sol before the aging or deposition steps. This enables proper passivation and functionalization of the dots to be performed in advance, thus resulting in highly photostable doped glasses [134]. Bullen and co-workers, for instances, have reported the successful immobilization of previously prepared QDs using colloidal methods [135]. In a second stage, the nanoparticles were capped with aminoethylaminopropyltrimethoxysilane (AEAPTMS) enabling their dissolution in polar solvents (such as propanol or ethanol). The capped nanoparticles were then added to the reacting mixture yielding a sol that could be used for deposition of thin films on different substrates ( $\mathrm{SiO}_{2}$ glass, soda-lime glass, silicon, polymers) by spin- or dipcoating techniques. The reported procedure allowed the homogenous dispersion of QDs in thin films without affecting their intrinsic emission properties. The authors also showed that the final films presented some waveguiding properties that depended on the type of immobilized nanocrystals. As the refractive index of the host matrix can be adjusted, this method is compatible with the realization of QD doped planar waveguide structures.

Although highly luminescent materials can be obtained by doping a sol-gel host with QDs, most of the reported applications are related with QD laser devices. To date the implementation of a chemical or biochemical sensor using sol-gel QD doped materials has been limited. Nevertheless, considering the variety of chemical and biological sensing applications using sol-gel immobilized organic dyes, such implementation would be realistic.

Conversely, a considerable number of polymer encapsulated QD sensing applications has been reported. A variety of materials and strategies have been addressed for chemical and biological sensing with polymer encapsulated QDs. Some of the more representative approaches were addressed in previous sections and include PMMA CdSe films for temperature sensing [91], acrylic nanospheres for QD ion sensing [101], a variety of polymer beads for imaging and labeling applications [121], QD MIP based polymers for selective sensing [103], among others. A summary of the most representative 
immobilized-QD sensing applications is given in Table 1. While a significant number of these applications used QD doped polymer micro- or nano-particles, and not bulk thin films, the application of the former can certainly be explored in composite materials amenable with coating techniques. This introduces the possibility of use in solid platforms such as fibers or planar waveguides.

Alternative immobilization approaches include the deposition of nanocrystals onto hydrophilic substrates using the Langmuir-Blodgett technique [136] or use of layer-by-layer (LbL) electrostatic self-assembly. The later technique is particularly attractive as it allows a very fine control of film thickness and deposition in surfaces of complex shape. In addition, it is compatible with nanocrystal functionalization and combination with further sensing dyes. Crisp and coworkers [137] have reported the coating of optical fibers and the inner surface of glass capillary with CdTe/ PDDA poly(diallyldimethyl-ammoniumchloride) - using LbL. Confocal microscopy data showed that the prepared coatings were found to be uniform, continuous and highly luminescent.

\subsection{Optical fiber sensing applications}

There are several techniques able to immobilize QDs, thus allowing the preparation of thin films displaying QDs' luminescent properties. While it is straight forward to obtain such luminescent materials, the type of solutions where QDs are simultaneously immobilized and allowed to interact with the environment for sensing purposes is still very limited.

In this context, it is no wonder that most optical fiber sensing schemes involving QDs are thermometry applications, where the only interaction requirement is the establishment of thermal equilibrium between the sensing membrane and the environment

Barmenkov et al. reported the first thermometry application using optical fibers and semiconductor nanocrystals [138]. In this work, the temperature dependence of the absorption band edge of CdSe QD-doped phosphate glass was used as the sensing mechanism. The CdSe doped glass plate $(6 \mathrm{~mm}$ thickness) was submitted to temperature changes while it was illuminated trough a standard multimode optical fiber using a white light source. The temperature dependent absorption spectra was then monitored using a second fiber and an optical spectrum analyzer. A spectral shift of the band edge of $0.12 \mathrm{~K} / \mathrm{nm}$ was reported. Using an HeNe laser to illuminate the sensor, the authors could translate the spectral shift into temperature dependent transmitted intensity, which was linear in the observed range. The operation range was of $0-150{ }^{\circ} \mathrm{C}$ due to limited thermal resistance of the polymeric fiber coating but could be extended up to $350{ }^{\circ} \mathrm{C}$ using special fibers. Because intensity measurements were performed, the application of this sensor in a practical application would be severely compromised by any source of optical power drift.

Table 1. Some representative examples of optical sensing with QDs immobilized in solid hosts. 


\begin{tabular}{|l|l|l|l|l|l|l|}
\hline QD-coating & Matrix & measurand & Mechanism & Features & platform & REF \\
\hline $\mathrm{CdSe}$ & $\begin{array}{l}\mathrm{SiO}_{2} \mathrm{Sol}-\mathrm{gel}, \\
\mathrm{PLMA}\end{array}$ & Temperature & $\begin{array}{l}\text { luminescence quenching and } \\
\text { bandgap shift; }\end{array}$ & $\begin{array}{l}100-315 \mathrm{~K}, \\
0.1 \mathrm{~nm} / \mathrm{K}\end{array}$ & glass slides & {$[91]$} \\
\hline $\mathrm{CdSe}$ & PMMA & $\begin{array}{l}\text { Methanol and } \\
\text { toluene }\end{array}$ & $\begin{array}{l}\text { luminescence quenching and } \\
\text { enhancement }\end{array}$ & Selectivity, PCA & glass slides & {$[98]$} \\
\hline $\mathrm{CdSe}-\mathrm{ZnS}$ & $\begin{array}{l}\text { Acrylic } \\
\text { (nanospheres) }\end{array}$ & $\mathrm{K}^{+}, \mathrm{Cl}^{-}$ & $\begin{array}{l}\text { FRET with chromophores and } \\
\text { luminophores }\end{array}$ & Dual sensing & $\begin{array}{l}\text { latex } \\
\text { microspheres }\end{array}$ & {$[101]$} \\
\hline $\mathrm{CdSe}-\mathrm{CdS}$ & $\mathrm{PVC} / \mathrm{DOS}$ & $\mathrm{Na}^{+}$ & lon exchange and energy transfer & Selectivity & microspheres & {$[102]$} \\
\hline $\mathrm{CdSe}-\mathrm{ZnS}$ & $\mathrm{MIP}$ & $\mathrm{Caffeine}$ & luminescence quenching; & Selectivity & $\begin{array}{l}\text { grinded } \\
\text { polymer }\end{array}$ & {$[103]$} \\
\hline $\mathrm{CdSe-ZnS}$ & polystyrene & IgG & $\begin{array}{l}\text { affinity binding with labelled } \\
\text { goat antihuman IgG }\end{array}$ & $\begin{array}{l}\text { QDs used for } \\
\text { color coding }\end{array}$ & $\begin{array}{l}\text { polymer } \\
\text { microbeads }\end{array}$ & {$[121]$} \\
\hline
\end{tabular}

Later on, a similar principle was used to provide an oxygen sensor with a temperature reference [139]. In this case, a colored glass filter (GG455 -cut-off $455 \mathrm{~nm}$ ) from Schott was placed at the distal end of one of the fiber tips of a 50/50 fiber coupler (multimode fiber with 550/600 $\mu \mathrm{m}$ core/cladding diameters). The filter cut-off wavelength was determined from the observed optical bandedge of CdSe QDs and it was shifted to higher wavelengths with increasing temperature by $0.08 \mathrm{~nm} /{ }^{\circ} \mathrm{C}$. The filter was chosen in order to maximize sensitivity by coinciding the cut-off wavelength with the spectral emission peak of the excitation source, a blue LED. When the blue radiation crossed the filter at the distal end of the fiber, it was possible to observe a temperature dependence on the back reflected intensity. In this case, a ratiometric scheme was implemented to avoid error induced by optical power drift. Because a fiber taper doped with an oxygen sensitive sol-gel glass was connected to the other fiber output of the coupler, this system enabled simultaneous oxygen and temperature measurements.

In the works mentioned above, the semiconductor nanocrystals have not been passivated and, therefore, the resulting composite material showed low luminescence at room temperature. In a recent work the use of luminescent colloidal nanocrystals for optical fiber temperature sensing has been reported [140]; distinct sized core-shell $\mathrm{CdSe} / \mathrm{ZnS}$ and $\mathrm{CdTe} / \mathrm{ZnS}$ QDs, with peak emission wavelengths at $520 \mathrm{~nm}, 600 \mathrm{~nm}$ and $680 \mathrm{~nm}\left(\mathrm{QD}_{520}, \mathrm{QD}_{600}, \mathrm{QD}_{680}\right)$ were immobilized using a nonhydrolytic sol-gel matrix [141]. The temperature behavior of different doped sol-gel samples was tested using an optical fiber bundle to excite and collect the nanocrystals' luminescent emission. A blue LED was used to excite all samples which were interrogated using a CCD spectrometer. The observed response was in accordance with the observation of Walker et al.[91]: as the temperature of the sensing samples was increased, the luminescence intensity decreased, the peak wavelength shifted towards the red and the spectral width increased. In the temperature range investigated, the changes were linear and reversible.

In Figure 6a the spectral response of a sample doped with $\mathrm{QD}_{520}$ can be observed. The temperature of the sample was increased from $14^{\circ} \mathrm{C}$ to $43^{\circ} \mathrm{C}$. From this data, it was possible to estimate that the luminescence intensity was decreased, as temperature increased, with a rate of $-1.6 \%$ per ${ }^{\circ} \mathrm{C}$. A similar behavior was obtained with other nanocrystals of different sizes with intensity decrease rates ranging between $-0.7 \%$ and $-1.6 \%$ per ${ }^{\circ} \mathrm{C}$. 

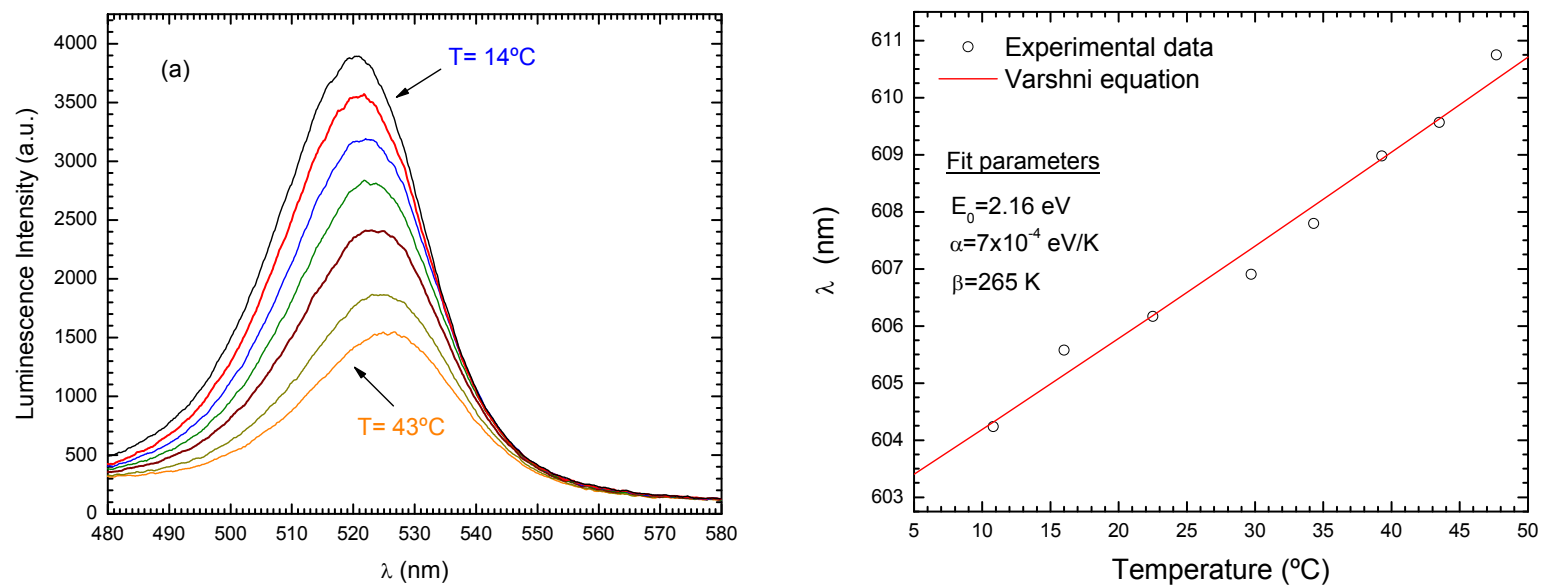

Figure 6. Temperature response of the luminescence emission of sol-gel immobilized $\mathrm{CdSe} / \mathrm{ZnS}$ nanocrystals for a range of $11^{\circ} \mathrm{C}$ to $48^{\circ} \mathrm{C}$ : a) Spectral response of $\mathrm{QD}_{520}$; b) Peak emission wavelength of $\mathrm{QD}_{600}$ as a function of temperature.

The peak wavelength of all samples, $\lambda_{\text {peak }}$, increased in a linear way as the temperature was raised. This is shown in Figure $6 \mathrm{~b}$ for a sample doped with $\mathrm{QD}_{600}$. The experimental data was well fitted to the Varshni relation which describes the temperature dependence of the band gap energy in the bulk semiconductor [142]. This indicates that the dominant process behind the temperature dependence of the luminescence peak in QDs is the same as in the bulk semiconductor and, therefore, it should be independent of the dot's size. In fact, as temperature increases, the band gap energy decreases because the crystal lattice expands and the inter-atomic bonds are weakened. As a consequence, less energy will be necessary to excite a charge carrier. On the other hand, it can be calculated that the change in the dot size due to thermal expansion has a negligible effect in the confinement energy. In addition, because the energy gap of both core and shell material change, although at different rates, the confinement energy change is still small when compared to the observed shift [143].

Comparing the wavelength shift in different samples it was concluded that for all the cases, the rate of change of $\lambda_{\text {peak }}$ towards longer wavelengths, as the temperature increased, was approximately 0.2 $\mathrm{nm} /{ }^{\circ} \mathrm{C}$.

Both luminescence intensity variation and the wavelength shift could be used to obtain temperature information. However, simple intensity measurements are prone to error due to optical power source fluctuations, detector drift, changing coupling conditions, etc. Therefore, the system susceptibility to optical power changes was evaluated. For this purpose the luminescent intensity response of $\mathrm{QD}_{600}$ to temperature was recorded for three different levels of LED output power $(100 \%, 90 \%$ and $80 \%)$. In Figure $7 \mathrm{a}$, it is clearly shown that the luminescence intensity response depended strongly on the LED output. Additionally, in each individual curve, a non-linearity was observed at lower temperatures, which was ascribed to water condensation at the surface of the sample which consequently changed the coupling conditions.

A simple detection scheme was implemented in order to take self-referenced temperature measurements. This was achieved by taking two signals, $S_{1}$ and $S_{2}$, corresponding to two narrow spectral windows on opposite sides of the spectrum, which were normalized according to 
$\mathrm{S}_{\mathrm{QD}}=\left(\mathrm{S}_{1}-\mathrm{S}_{2}\right) /\left(\mathrm{S}_{1}+\mathrm{S}_{2}\right)$. Due to the presence of a temperature dependent wavelength shift, the resulting output was proportional to temperature and independent of the optical power level in the system. With the application of this scheme, the temperature measurements were rendered independent of the optical power level (shown in Figure $7 \mathrm{~b}$ ). An accuracy of approximately $0.3{ }^{\circ} \mathrm{C}$ was estimated by linear regression analysis.
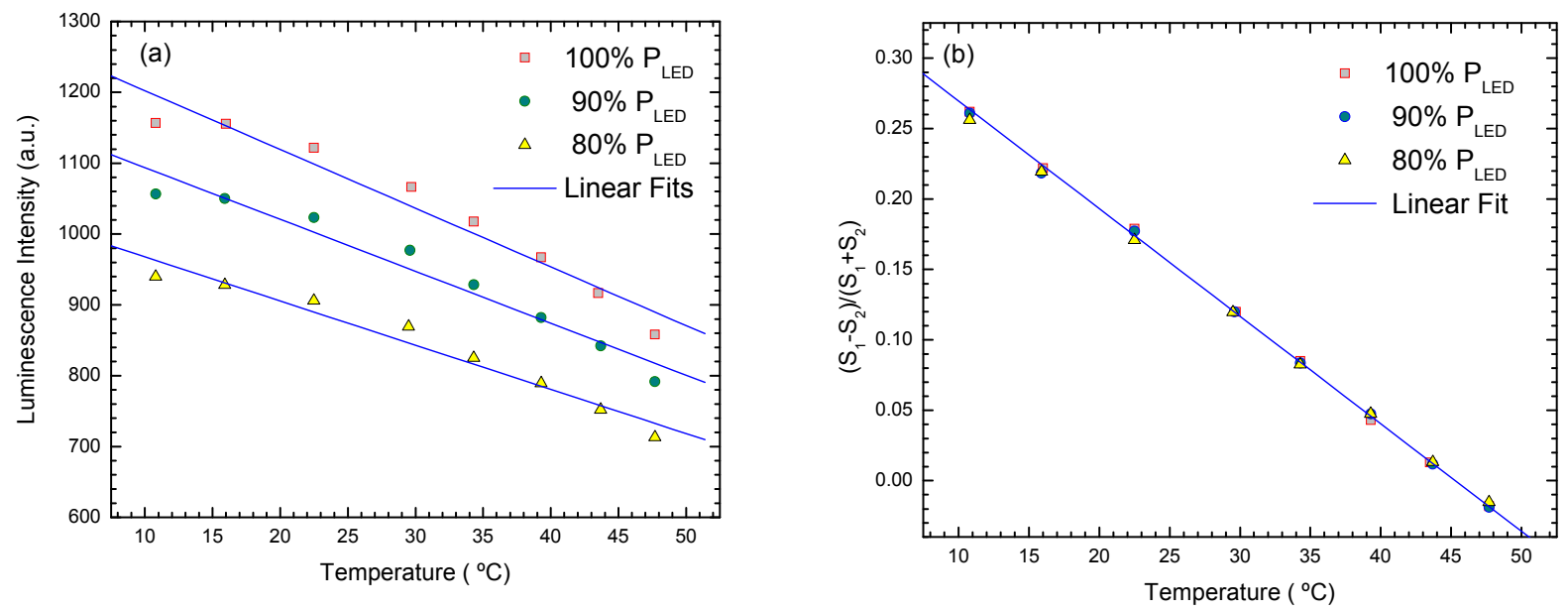

Figure 7. a) Luminescent intensity of $\mathrm{QD}_{600}$ as a function of temperature for three levels of LED optical power $(100 \%, 90 \%$, and $80 \%)$; b) Corresponding normalized outputs $\left(\mathrm{S}_{\mathrm{QD}}\right)$.

These results demonstrated that QDs can be used as self-referenced temperature probes. The ratiometric processing is made possible by the presence of a wavelength shift and can be a valuable tool in many applications since, most of the times, temperature is an important parameter for any biological system.

The same detection scheme was used to simultaneously monitor two samples doped with distinct QDs using the same optical fiber system. Both transmission and reflection topologies were successfully tested, using optical fiber bundles (shown in Figure 8), demonstrating the QDs' potential for multiplexed optical fiber sensing. Figure 9a shows the spectral response of two different samples $\left(\mathrm{QD}_{600}\right.$ and $\left.\mathrm{QD}_{680}\right)$ displaying well discriminated emission spectra with independent temperature responses. This can be further confirmed observing the simultaneous response of both samples as their temperature changed independently (Figure 9b). To the best of our knowledge this was the first optical fiber multiplexing application using QDs [140].

In a different approach, De Bastida et al. used the LbL technique to coat a tapered optical fibre tip with CdTe QDs of different sizes [144]. The produced fiber probes were then used as luminescent thermometers. The behaviour of the dots luminescence intensity and wavelength was very similar to those reported by other authors, which demonstrated the preservation of the QDs' luminescent features. Nevertheless, because no core-shell QDs were used, thermal annealing under a nitrogen atmosphere was carried out in order to increase the resistance of QDs towards oxidation. In another work, the same authors demonstrated the feasibility of coating the inner surface of hollow core fibers with a film composed of QDs [145]. The fiber inner diameter was $50 \mu \mathrm{m}$ demonstrating the ability of 
the LbL technique for coating morphological complex surfaces of reduced dimensions. The produced tips were used as a temperature sensor.

While this was the first reported LbL QDs sensor, the versatility of this technique will contribute for the feasibility of further biochemical optical fiber QD-based sensing applications. A representative example of the versatility of a QD-LbL combination was reported by Ruan et al.. In this work, the authors took advantage of the semiconductor properties of CdSe/ZnS QDs in order to assemble a photodetector on the surface of an optical fiber tip using the LbL technique [146]. This demonstration allows one to envision future applications where QDs can simultaneously fulfill the role of an optical source, a detector and the sensing layer.

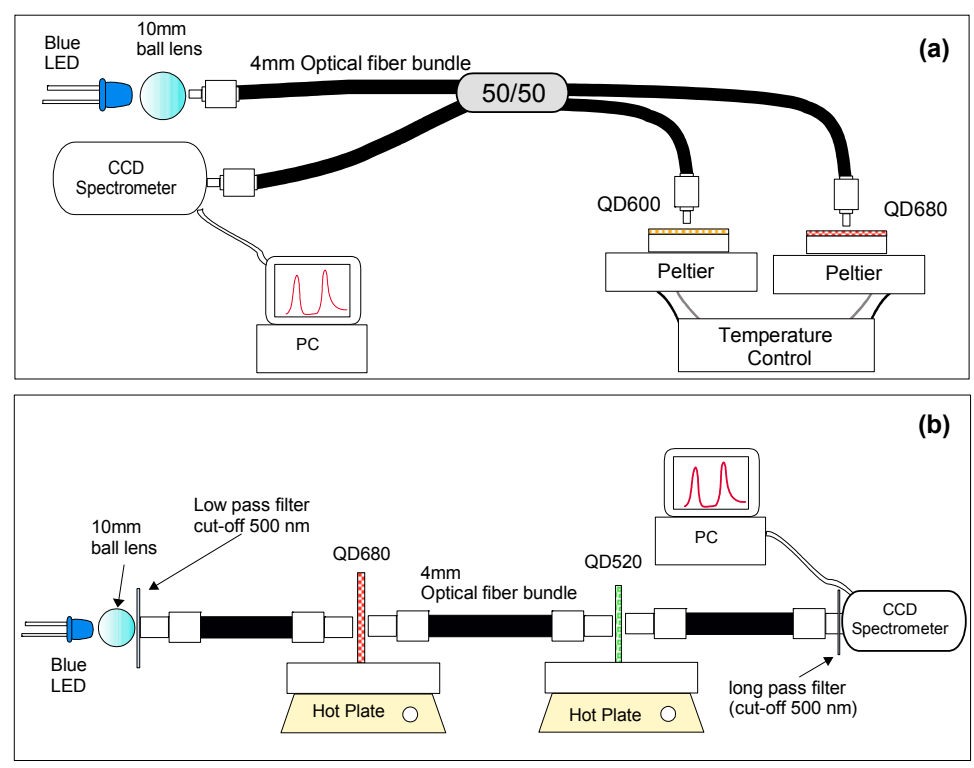

Figure 8. Reflection (a) and transmission (b) configurations used to interrogate simultaneously two different samples of sol-gel glass doped with semiconductor nanocrystals.

Temperature is of paramount importance in many biochemical sensing applications because the luminescent response of organic dyes always depends on this working parameter. Therefore, combining nanocrystals with a sensing dye, it is possible to obtain simultaneous information about a chemical parameter and temperature. Due to the ability to tune their optical properties, QDs with no spectral overlap with a particular sensing dye can be easily chosen allowing this technique to be implemented in a variety of applications.

Recently, the application of QDs in a dual sensing configuration was demonstrated in the context of an oxygen sensor [147]. Temperature has a double effect on the sensor calibration function. It introduces higher probability of non-radiative transitions, thus decreasing the luminescence yield and the lifetime of the excited state, and it changes the oxygen diffusion coefficient into the sensing membranes. In order to obtain an univocal measurement of oxygen concentration by luminescent methods the simultaneous determination of temperature is required. CdSe/ZnS QDs were used to provide such independent measurement. The nanocrystals were immobilized in a dense non-hydrolytic $\mathrm{SiO} 2$ sol-gel material, with low oxygen permeability, presenting an oxygen independent luminescence output. Also, QDs emitting at $520 \mathrm{~nm}$ were used to minimize any spectral overlap with the excitation source (a blue LED - $473 \mathrm{~nm}$ ) or the oxygen sensitive luminescence signal (600 $\mathrm{nm}$ emission from 
Tris(4,7-diphenyl-1,10-phenanthrolin) ruthenium (II) chloride -Ru(dpp)-). An optical fiber coupler $(600 / 550 \mu \mathrm{m}$ silica fiber) was used to excite and interrogate the QDs sample together with an oxygen sensing sample (sol-gel doped with $\mathrm{Ru}(\mathrm{dpp})$ ).
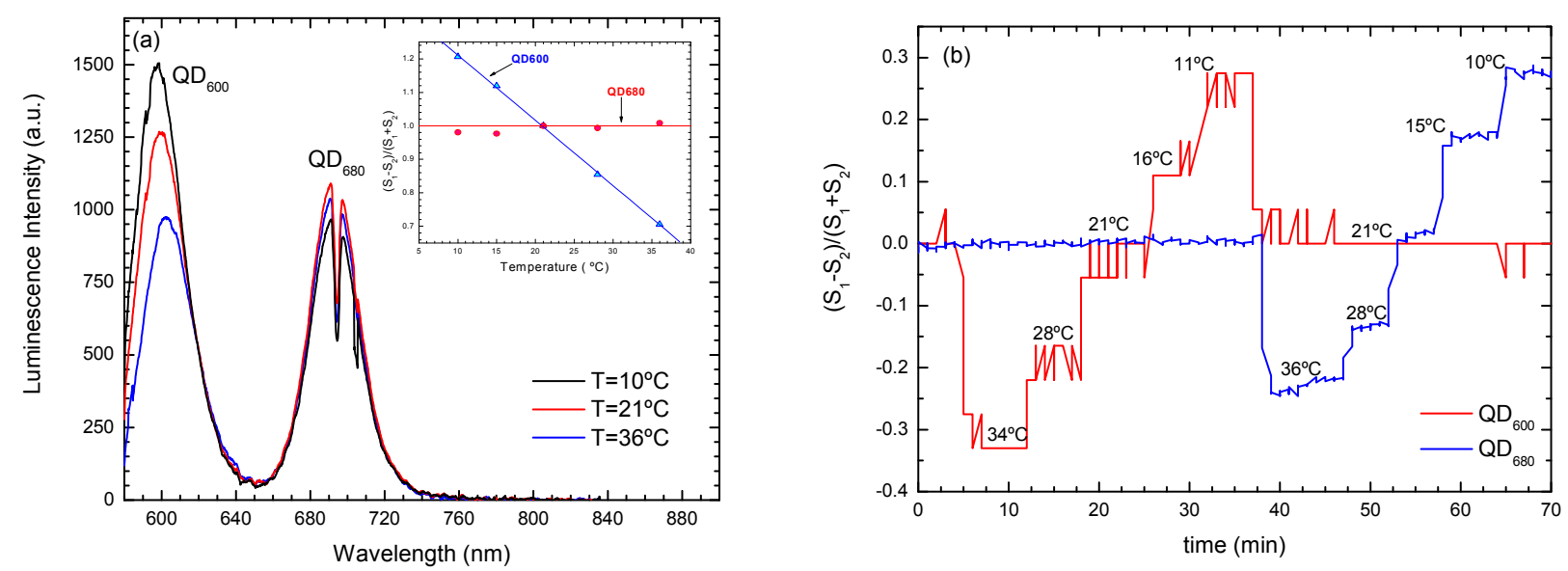

Figure 9. a) Spectral response of two distinct $\mathrm{QD}$ samples $\left(\mathrm{QD}_{600}\right.$ and $\left.\mathrm{QD}_{680}\right)$ to independent changes of temperature (inserted: Normalized $\mathrm{S}_{\mathrm{QD}}$ signals for both samples as function of the applied temperature - only the temperature of $\mathrm{QD}_{600}$ was changed); b) Normalized responses, $\mathrm{S}_{\mathrm{QD} 600}$ and $\mathrm{SQ}_{\mathrm{D} 680}$ during a time interval in which alternate independent temperature changes were applied to each sample.

Each of the sensing elements was individually calibrated and it was shown that oxygen measurements could be made with an uncertainty of $\pm 2 \%$ for oxygen concentration, while for temperature this range was $\pm 1^{\circ} \mathrm{C}$. This poor performance, as compared to previous reports using single parameter sensing, was mainly due to a reduced signal-to-noise-ratio (SNR). LED excitation together with the low efficiency of extrinsic sensing elements contributed to this problem. Moreover, it was possible to demonstrate dual parameter sensing using this scheme. In Figure 10, the response of the two sensors to applied temperature and oxygen changes can be observed. The sensors were submitted to alternate atmospheres of air and pure nitrogen while a temperature step was applied.

Figure 10a shows the oxygen measurements obtained directly from the $\mathrm{Ru}(\mathrm{dpp})$ luminescent output. It is shown that the luminescence intensity followed the changes in oxygen content. However, while at ambient temperature it accurately yielded concentrations of nearly $0 \%$ (in nitrogen) and $21 \%$ (in air), at an higher temperature the retrieved values were of $10 \%$ (in nitrogen) and $30 \%$ (in air). The increased concentration values resulted from a temperature induced error.

The QDs output, on the other hand, allowed to accurately retrieve the applied temperature step, showing no sensitivity to the changes in oxygen concentration (Figure 10b). Since the temperature response of $\mathrm{Ru}(\mathrm{dpp})$ was known, using the temperature information from the QDs, it was possible to correct the oxygen measurements. Figure 10c shows that the corrected response was accurate throughout the whole temperature range. Therefore, a $10 \%$ error in oxygen concentration, induced by a temperature variation of a few degrees, could be fully compensated using the dual sensing scheme. 

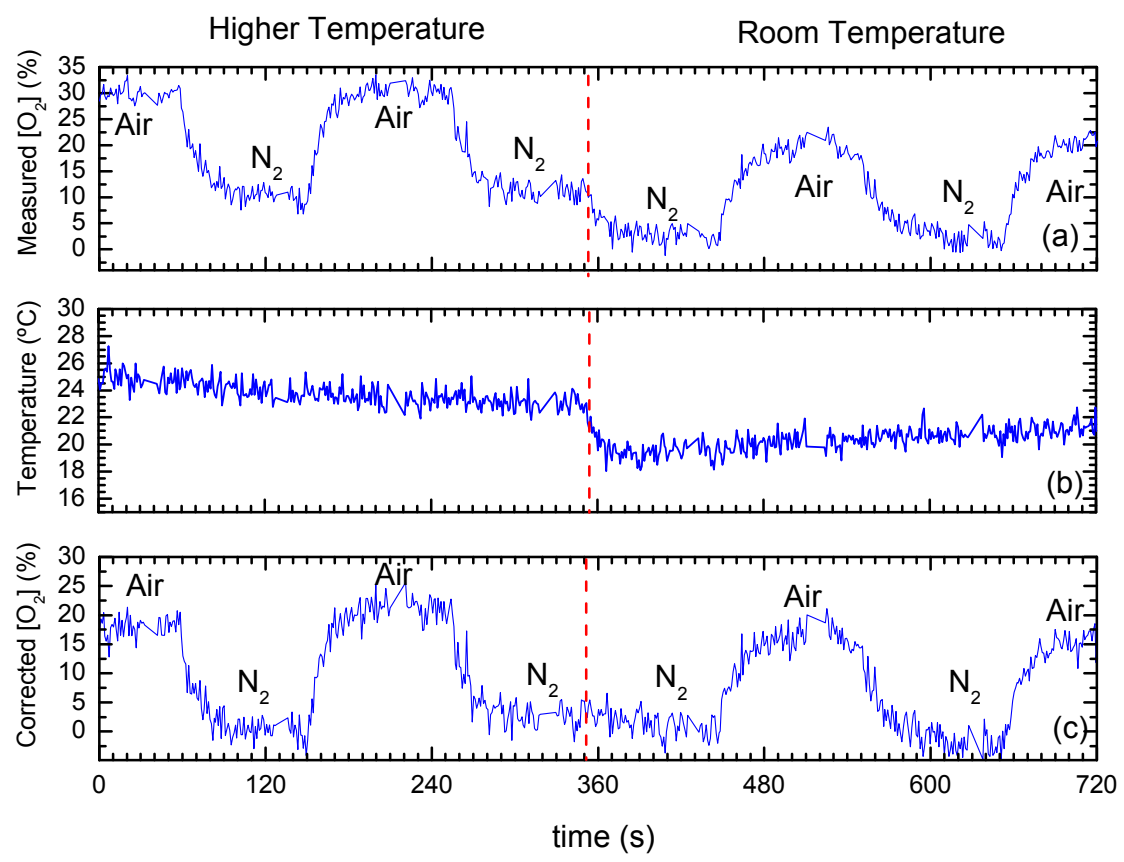

Figure 10. Sensor output when subjected to simultaneous change of oxygen and temperature: (a) $\mathrm{O}_{2}$ measured by $\mathrm{Ru}\left(\mathrm{dpp}\right.$ ); (b) temperature measured by QD; (b) temperature compensated $\mathrm{O}_{2}$ measurement.

While the results obtained where not impressive from the accuracy point of view, there is much room for improvement. Using intrinsic fiber probes, associated with laser excitation, can allow to increase the SNR and, therefore, the precision of the measurements. The QDs' tuneability, on the other hand, will enable the application of this scheme to virtually any luminescence based sensor.

Other possibilities of QDs improving the performance of oxygen sensors were recently addressed [148]. By using a sol-gel prepared $\mathrm{SiO}_{2}$ sample doped with $\mathrm{CdSe} / \mathrm{ZnS}$ QDs, with low oxygen permeability, combined with an oxygen sensing film, it was possible to implement a ratiometric reference scheme. In this particular case, temperature was kept constant and the luminescent output of $\mathrm{QD}_{520}$ was used as an intensity reference. The oxygen sensor was obtained by doping sol-gel glasses with the complex $\left[\mathrm{Ru}(\mathrm{bpy})_{3}\right]^{2+}$ (bpy: 2,2'-bipyridine). Only wavelengths lower than $520 \mathrm{~nm}$ are absorbed by these nanocrystals. Therefore, its luminescent intensity was affected by the excitation radiation but not by the emission of ruthenium dyes. As a consequence, oxygen had no effect on the QDs' emission, which was proportional only to the exciting optical power. From these conditions it resulted that the ratiometric detection of the luminescence emissions of QDs and a $\left[\mathrm{Ru}(\mathrm{bpy})_{3}\right]^{2+}$ doped sensor were proportional to oxygen and independent of the optical source intensity. This is clearly shown in Figure 11a where the ratiometric output is compared with the raw $\left[\mathrm{Ru}(\mathrm{bpy})_{3}\right]^{2+}$ emission while the luminescence output of the excitation source is slowly modulated.

Using this scheme it was possible to compensate intensity changes of the excitation source up to $80 \%$. In principle, it would be possible to use this scheme even with changing temperatures. Temperature would be given by the wavelength shift and used to correct the intensities of both QDs and the oxygen sensor. The corrected QDs intensity could then be used as an intensity reference. In spite of all, the scheme does not eliminate the calibration drift due to photobleaching. 

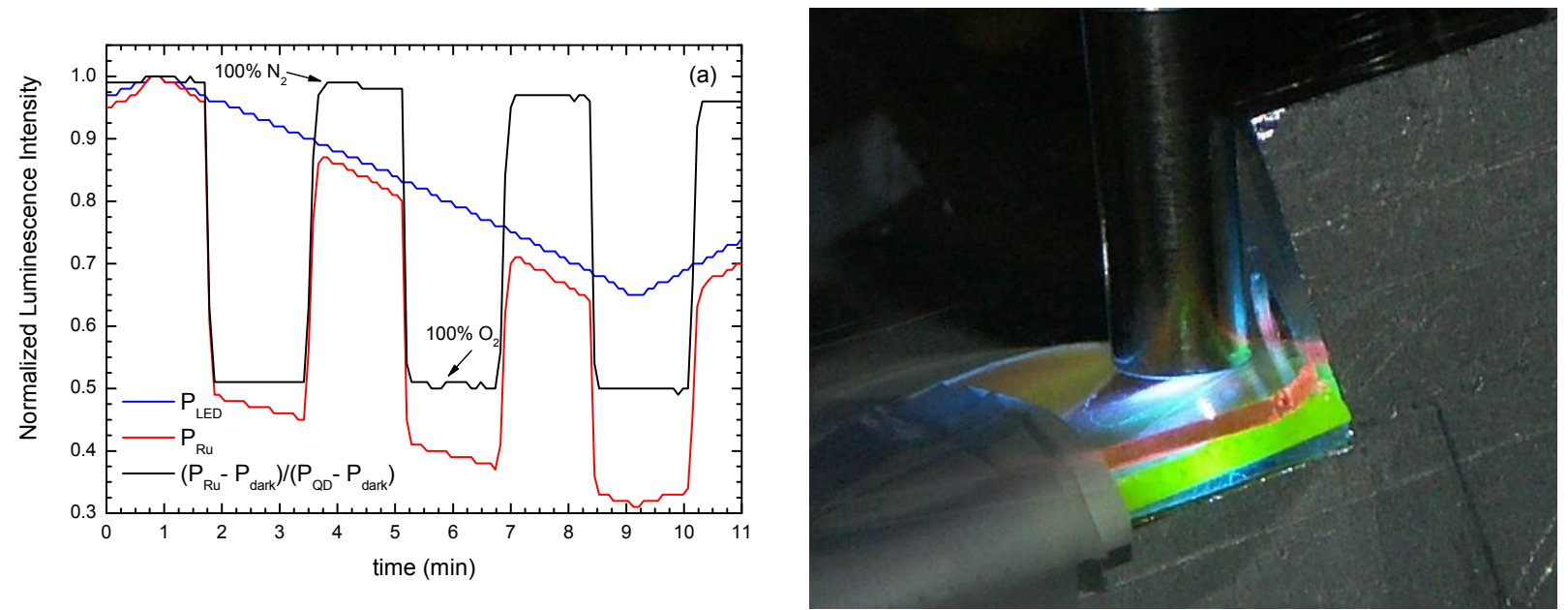

Figure 11. a) Sensor response to $\mathrm{O}_{2} / \mathrm{N}_{2}$ saturation cycles while excitation optical power was slowly changed from $100 \%$ to $70 \%$. Both the raw luminescent output signal of $\mathrm{Ru}(\mathrm{bpy})$, and the ratiometric signal obtained using the QDs luminescence are shown. b) a picture of the luminescent QD and oxygen sensing samples excited using a fiber bundle.

Using QDs with an emission wavelength shorter than that of an associated sensing dye, will provide a variety of luminescence based biochemical sensors with very stable intensity or temperature references. Conversely, if the QD's emission is shifted towards the red, using an emission wavelength higher than that of the sensing dye will introduce the possibility of transforming the QDs in a sensing indicator.

The possibility of obtaining oxygen sensors with different spectral signatures was investigated. $\mathrm{CdTe} / \mathrm{ZnS}$, with emission peak at $680 \mathrm{~nm}$, were immobilized in sol-gel glass and sandwiched together with a $\mathrm{Ru}(\mathrm{dpp})$ doped sol-gel sample, as shown in the insert of Figure 12b, and placed inside a closed chamber. To avoid direct excitation of the QDs from the LED, a long pass (600 nm cut-off) filter was placed between the two samples. Excitation and detection were performed using fiber bundles.

Figure 12b shows the spectral response of the sandwich arrangement to atmospheres of pure oxygen and pure nitrogen. The response of a film of $\left[\mathrm{Ru}(\mathrm{bpy})_{3}\right]^{2+}$ alone is shown in Figure 12a for comparison purposes. The emission peak corresponding to the nanocrystals emission is clearly visible in Figure 12b. In addition, some features appear in the spectral region of 650-675 nm, looking like a secondary peak or a depression in the dye emission, which could be due to some wavelength dependent absorption of the dye radiation by the QDs. The emission of the nanocrystals is clearly oxygen dependent.

A careful comparison between the spectral responses with and without the QDs allowed to confirm an increase in the sensitivity to oxygen in the spectral region between 700-800 nm. Maximum enhancement took place at approximately $760 \mathrm{~nm}$. At this particular wavelength, the quenching efficiency was improved by a factor of 2.4 .

These results demonstrate that it is possible to obtain oxygen sensitivity in different spectral regions using QDs. The ideal situation would be to have nanocrystals emitting in the near infrared with no spectral overlap with the ruthenium dyes. The application of this principle using nanocrystals with 
different emission peaks, combined with the adequate sensing dye, would allow to obtain a set of oxygen sensors with different spectral signatures, suitable for wavelength multiplexing. Nanocrystals with longer emission wavelengths would greatly enhance the performance of this sandwich configuration since their emission spectra would not overlap with that of the sensing dye. In principle, this could be achieved using InAs or PbSe QDs, providing interesting solutions for near infrared wavelength multiplexed chemical sensing. In a more sophisticated approach, FRET could be used if the QDs and the indicator were adequately conjugated. However, the major problem of avoiding direct excitation of the sensing dye by the QDs would still limit the sensor performance.
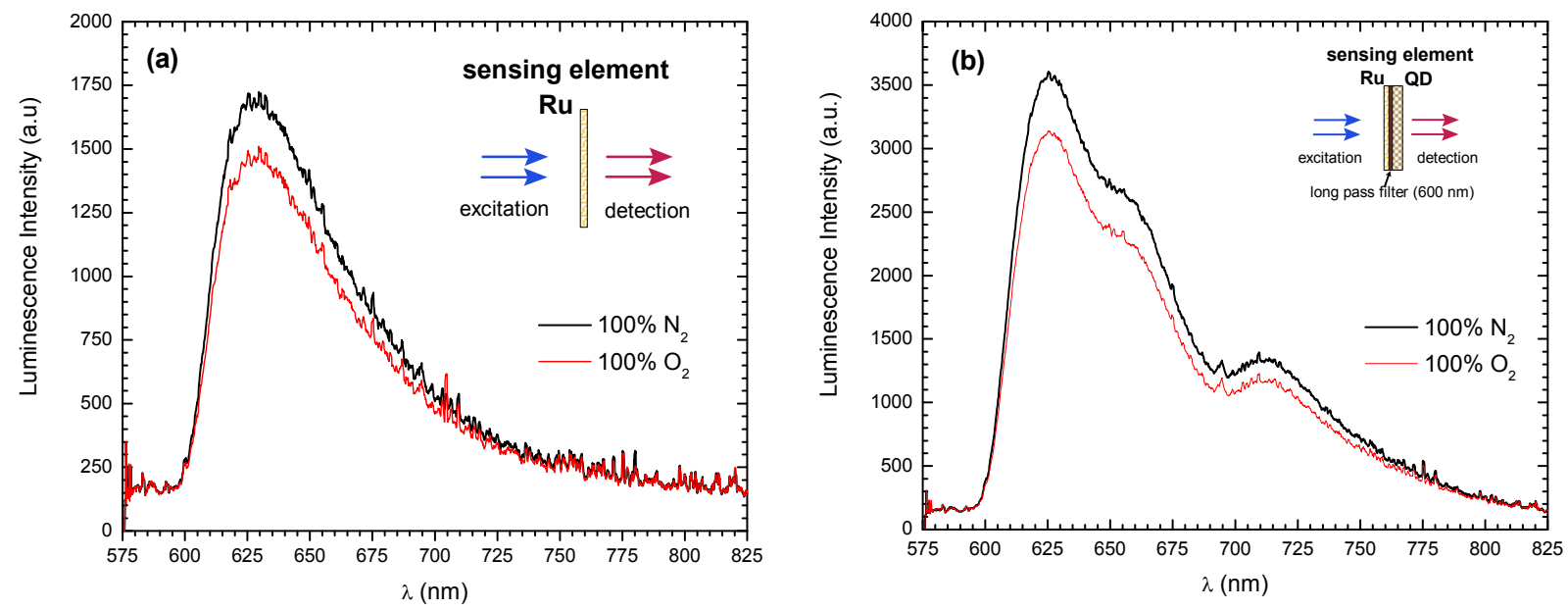

Figure 12. Spectral responses in saturated atmospheres of $\mathrm{N}_{2}$ an $\mathrm{O}_{2}$ (Inserted: a scheme of the sensing configuration): (a) Ru(bpy); (b) Ru(bpy) + long pass filter (600 nm) + $\mathrm{QD}_{680}$.

In what was probably the first QD-based optical fiber biosensor, a reagentless, regenerable and portable immunosensor was developed by Aoyagi and Kudo [149]. Qdot655 ${ }^{\mathrm{TM}}$-labeled proteinA, purchased from Quantum Dot Corporation, were immobilized on the surface of a $1 \mathrm{~mm}$ diameter glass slide. The functionalized glass membrane was them placed on top of an Y fiber bundle through which excitation and detection of luminescent signals could be performed using a fluorometer (Figure 13a).

The sensing probe was then used to selectively detect immunoglobulin G (IgG) in standard solutions containing other proteins. It was shown that the binding of IgG to proteinA caused quenching of the QDs luminescence intensity due to FRET between the nanocrystals and the bound sample protein. The quenching rate was proportional to the immunoglobulin concentration in the 0.0 to 6.0 $\mathrm{mg} / \mathrm{mL}$ as it can be seen in Figure 13b. The measuring range could be modified by changing parameters such as the amount of immobilized protein A on the glass plate and the diameter of the detecting optical fiber. Although 20 min were necessary for luminescence to attain the steady state, the authors observed the dynamics of the quenching process and concluded that most of the quenching events took place within the first minute of reaction, allowing for nearly real time detection. The sensing probes could be regenerated as the IgG bonds to protein A can be broken in a low $\mathrm{pH}$ solution ( $\mathrm{pH} 2-4)$. This pioneer work demonstrated that QDs can be used to develop biosensing probes with very attractive characteristics such as selectivity, reversibility and in-situ operation with no need to add extra reagents to the probed solutions. 
Besides the LbL configurations, all the sensing schemes reported so far were extrinsic probe configurations. Microstructured optical fibers (MOF), such as photonic crystal fibers (PCF) or holey fibers, promise to be excellent platforms for the development of intrinsic fiber probes. Particularly, some of these fibers typically rely on an array of air-filled tubular structures surrounding the nucleus to provide guiding with an high index contrast. Besides giving the fiber superior guiding properties, these holes can be filled with doping materials which can be evanescently excited. These holey structures have a great potential for sensing applications that is just starting to unveil.
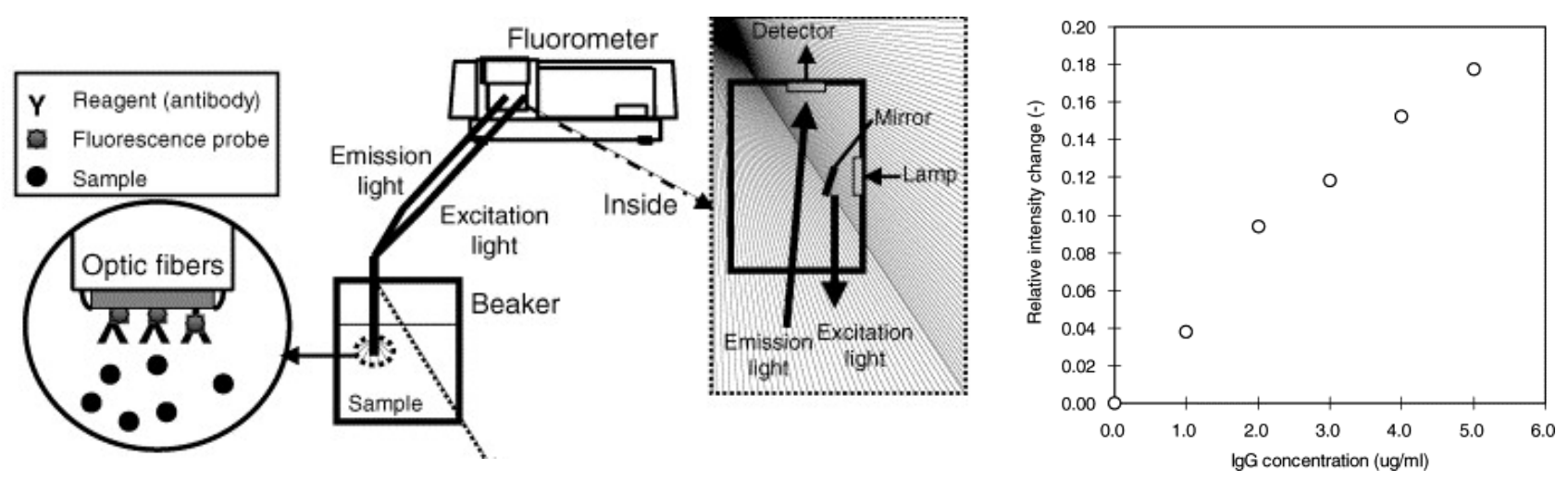

Figure 13. a) Schematic diagram of the reagent-less fiber-optic fluorescent immunosensor. b) Typical relationship between the IgG concentration and the fluorescence intensity change of QD-protein A on a glass plate [149]. Reproduced from Biosensors and Bioelectronics, 20, Satoka Aoyagi and Masahiro Kudo, Development of fluorescence change-based, reagent-less optic immunosensor, 1680-1684, Copyright (2005), with permission from Elsevier.

In what concerns combination of MOF with QDs, some reports have been made where the nanocrystals were used to dope the fiber's voids. While the aim in these particular works was to use QDs as a gain medium for optical fiber lasers, the techniques that were developed can be applied in sensing devices. Meissner et al. have recently studied the behavior of CdSe-ZnS nanocrystals entrained in an array of $14.6 \mu \mathrm{m}$ holes surrounding the $12.7 \mu \mathrm{m}$ core of a MOF [150]. Pieces of approximately $10 \mathrm{~cm}$ length were immersed in a colloidal suspension of $573 \mathrm{~nm}$ emitting nanocrystals in heptane. A 5 min immersion was enough to fill the holey structure with the QDs solution by capillarity. The doped fibers were then pumped with a $488 \mathrm{~nm}$ argon line and probed with a $594 \mathrm{~nm} \mathrm{HeNe}$ laser propagating through the fiber core.

Using this method, the authors were able to excite the QDs luminescence which was then coupled into the fiber core and guided. In addition, it was observed that the amount of probe light observed from the end of the fiber was increased when the pump and probe were both present. The authors suggested that the extra light appearing in both the fiber core as well as the outer, solid clad region, could be caused by optical gain. This claim, however, was a subject of debate because, typically, high rate pulsed pumping is necessary for QD gain to take place [151, 152].

In short term observations the QDs' emission was shown to be preserved, however, slow degradation of the entrapped solution in the long term is a concern. In a more fundamental approach, $\mathrm{Yu}$ and coworkers have developed a versatile method for doping polymer MOF [153]. The authors where able to dope the core of micro-structured plastic optical fiber (POF) with CdSe-ZnS 
nanocrystals. This was achieved by acting prior to the drawing process. A polymeric rod doped with QDs was inserted into the central hole of an intermediate perform with an $11 \mathrm{~mm}$ external diameter. After the drawing process a suspended-core fiber with outer and core diameters of $400 \mu \mathrm{m}$ and 130 $\mu \mathrm{m}$, respectively, was obtained. Because the QDs were in the fiber core a more efficient excitation and guiding capability could be obtained. The authors suggested that due to the relatively low processing temperatures employed $\left(\sim 200{ }^{\circ} \mathrm{C}\right)$ this method will enable the incorporation of both organic and inorganic materials. In this context, although the primary goal of the authors was the development of QD based optical sources and switches, this method was an important step towards the feasibility of MOF based biochemical sensors.

The possibility of implementing intrinsic fiber sensors using PCF was investigated using organic luminescent dyes and QDs as doping agents [154]. The fiber used was an endlessly single mode PCF type ESM-12-01 with 54 microchannels of $6.2 \mu \mathrm{m}$ diameter separated by $8.0 \mu \mathrm{m}$ spacing. The holey structure of small pieces of PCF (typically $20 \mathrm{~mm}$ ) were filled, by capillarity, with different quantum dots, dissolved in toluene or mesitylene at micromolar concentrations.

The fluorescence emission of the QDs inside the PCF was then observed using a microscope with adequate filtering to reject the excitation light at $365 \mathrm{~nm}$. The cross section of the doped fiber is shown in Figure 14A where the QDs green luminescence is clearly visible.

In order to evaluate the possibility of detecting free radical species, the doped fiber tips were exposed to a $0.5 \mathrm{M}$ toluene solution of TEMPO (2,2,6,6-tetramethylpiperidine-N-oxide free radical). This oxidative species was shown to strongly quench the luminescence of the QDs in solution (Figure 14C). The addition of the TEMPO solution to the fiber tip lead to an almost immediate quenching of the observed luminescence in the immersed tip, while the opposite end (20 $\mathrm{mm}$ away) remained fluorescent with little immediate change of emission intensity. As shown in Figure 14B, the fluorescence intensity of the sensing tip was then almost totally recovered after a few minutes, remaining practically unchanged for at least a week. The recovery of the luminescence was attributed to the high non-linearity of the quenching of QDs' emission by paramagnetic species combined with the diffusion/dilution of the analyte into the fiber. This was confirmed by observing that larger QDs, emitting in the red, displayed a more linear quenching behavior, because of their lower diffusion coefficient. This way, the recovery of luminescence was very poor and much slower when using larger nanoparticles.

The authors suggested that QDs could alternatively be incorporated in a polymer matrix coating the inner surface of the micro channels. This would enable potentially reversible sensing tips to probe different sample solutions without loosing the indicator. Although the potential of MOFs for biosensors is being explored by different authors a fundamental difficulty arises from the need for analyte solution to flow through the fiber microstructure. This precludes the fusion of the MOF tips in standard fiber interrogation systems. Nevertheless, recent works have been presented that demonstrated the possibility of incorporating MOF into microfluidic circuits for use in biochip applications $[155,156]$. 

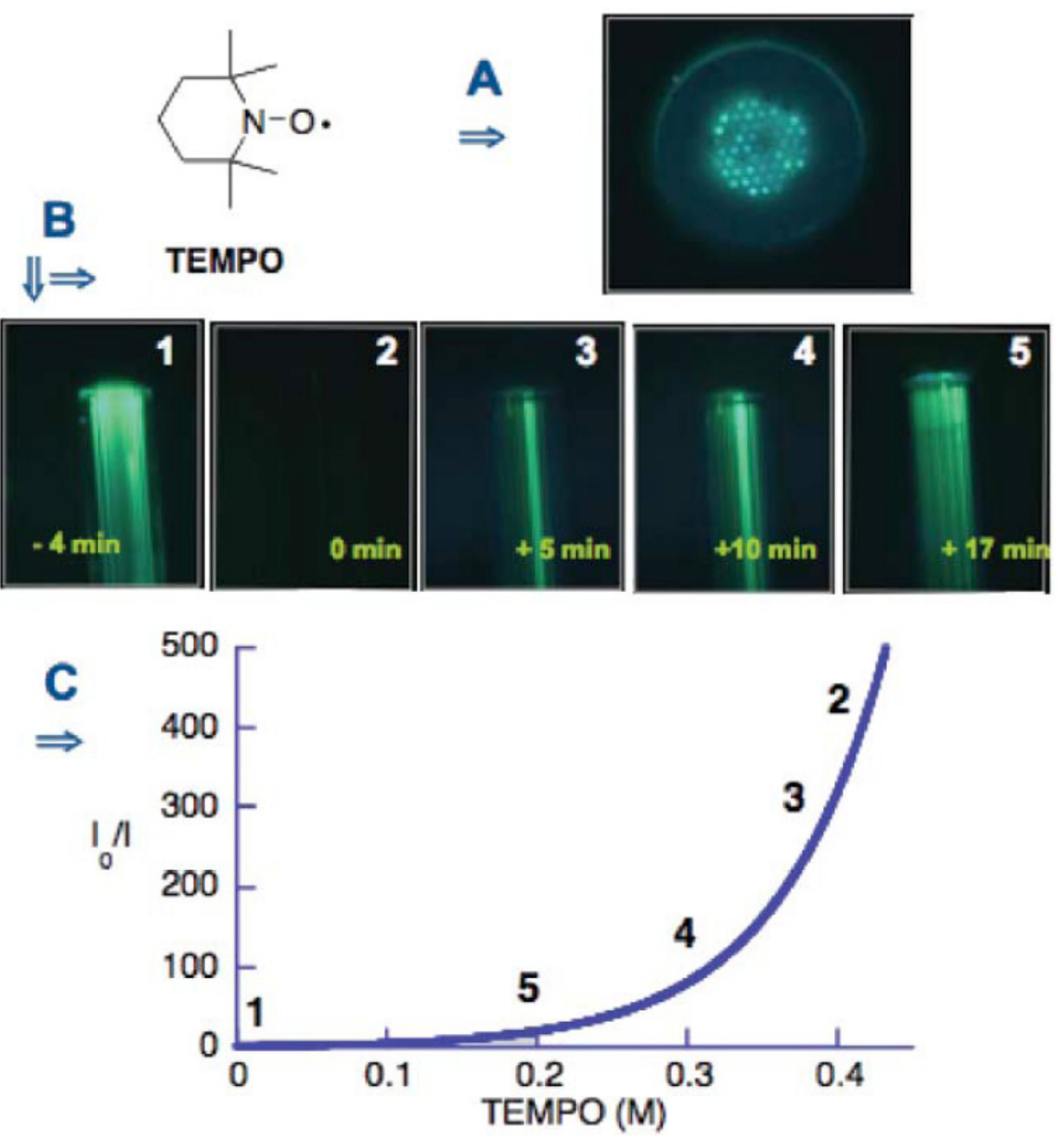

Figure 14. (A) Fluorescence photograph of the PCF end containing green QDs (2.4 nm) after addition of TEMPO and recovery. (B) Time lapse fluorescence photographs (from left to right) before adding TEMPO, immediately after, 5, 10, and 17 minutes after addition. Photographs were taken in normal (A) and transverse (B) mode. Small color differences with angle are common in photonic crystals. (C) Stern Volmer plot for quenching of green QD by TEMPO. The numbers in (C) indicate approximately the delay following addition, with ' 1 ' corresponding to data before TEMPO addition. [154] Reproduced by permission of The Royal Society of Chemistry.

A summary of the applications where QDs were applied in optical fiber sensors is given in Table 2. Although, to date, very few applications on the use of nanocrystals in fiber sensors have been reported, some of the works presented were key steps towards the development of new sensing tools. QDs immobilized in different matrix were used in a diversity of applications from thermometry to biosensing demonstrating that the unique features of QDs together with its physicochemical versatility will soon introduce a new class of advanced analytical tools. 


\subsection{Applications of $Q D$ s in planar structures}

Planar structures using waveguide or microarray configurations are very appealing platforms that, when combined with microfluidics and electronics, can configure Lab-on-a-Chip (LOC) devices. LOC systems offer the possibility to carry out different functions, such as sample preparation, concentration and detection in a single miniaturized platform that has strong potential for batch analysis purposes. While LOC technologies have seen some impressive progresses [157-159], the application of QDs in this field is still small. Nevertheless, QD properties are very appealing for such applications, particularly because of their high photostability, multicolor ability and increased equivalent Stokes shift as these characteristics allow high density detection with increased noise rejection.

Besides the immobilization methods previously described, some patterning and manipulation techniques need to be developed for the feasibility of QD based LOC devices.

In a very interesting approach, QD encoded polymer microbeads where manipulated trough the use of a microfluidic Lab-on-a-disk structure [160]. The combination of centrifugal forces together with microfluidic channels and a geometrical barrier allowed to aggregate the color-encoded beads in a monolayer within a disk-based detection chamber. A parallel read out scheme could then be implemented using a color CCD-camera. The automated localization, color identification, and fluorescent detection aiming for color-multiplexed fluorescence immunoassays was made possible by a dedicated image processing software. The authors could successfully identify three distinct encoded microbeads, using two types of QDs and an organic luminophore, thus demonstrating multiplexing ability. In a practical situation, each color-code can identify polymer beads functionalized with different antibodies, allowing for multiplexed imunodetection using standard fluorescence immunoassay techniques. The viability of the detection system was successfully demonstrated by performing a hepatitis A and a tetanus assays on the microfluidic lab-on-a-disk platform.

In order to avoid systems with moving parts and readout schemes with complex signal processing, the nanocrystals should be immobilized on a solid subtract. In such cases, patterning strategies are necessary for the implementation of multiparameter devices.

Lithographic techniques have been adapted to implement spatially separated patterns of different colloidal nanocrystals in an aminofunctionalized substrate [161]. By using masks and exposure with UV light, different sites could be bounded to the substrate surface in a selective mode allowing to obtain well defined pixilated microarrays. Similar patterning could be obtained using contact masking and ion implantation techniques [162]. Also by functionalizing the nanocrystals surfaces with either hydrophobic or hydrophilic ligands, as discussed previously, they can bind to selected sites on the substrate forming dual color arrays [163]. More recently, using the sol-gel technique, QDs patterns were formed by using UV assisted photosynthesis [164]. Using this method a precursor solution was spin coated on a subtract and illuminated by a patterned UV beam. Because the photochemical reactions only took place on the illuminated sites, an ordered array of QD pixels with diameters of few microns could be formed. Reshaping the curing UV beam, patterns could be made in a range of sizes up to hundreds of microns. 
Table 2. - QD applications in optical fiber sensing.

\begin{tabular}{|c|c|c|c|c|c|c|c|}
\hline QD-coating & Matrix & Measurand & Mechanism & Features & $\begin{array}{l}\text { Fiber } \\
\text { platform }\end{array}$ & probe & REF \\
\hline $\mathrm{CdSe}$ & $\begin{array}{l}\text { Phosphate } \\
\text { glass }\end{array}$ & Temperature & bandgap shift - absorption & $\begin{array}{l}0-150^{\circ} \mathrm{C}, \quad 0.12 \\
\mathrm{~nm} / \mathrm{K}\end{array}$ & multimode & extrinsic & [138] \\
\hline $\mathrm{CdSe}$ & $\begin{array}{l}\text { Schott glass } \\
\text { filter }\end{array}$ & Temperature & bandgap shift - absorption & $\begin{array}{l}\text { Simultaneous } \\
\text { detection } \mathrm{O}_{2} \text { and } \\
\text { temperature }\end{array}$ & multimode & extrinsic & [139] \\
\hline $\begin{array}{l}\text { CdSe-ZnS-TOPO; } \\
\text { CdTe-ZnS }\end{array}$ & Sol-gel & Temperature & $\begin{array}{l}\text { luminescence quenching and } \\
\text { bandgap shift; }\end{array}$ & $\begin{array}{l}\text { Self referenced } \\
\text { Multiplexed }\end{array}$ & fiber bundle & extrinsic & {$[140]$} \\
\hline CdTe- PDDA & $\mathrm{LbL}$ & Temperature & luminescence quenching; & $\begin{array}{l}30-100^{\circ} \mathrm{C}, \quad 0,2 \\
\mathrm{~nm} /{ }^{\circ} \mathrm{C}\end{array}$ & $\begin{array}{l}\text { Multimode/ } \\
\text { tapered }\end{array}$ & intrinsic & {$[144]$} \\
\hline CdTe- PDDA & $\mathrm{LbL}$ & Temperature & luminescence quenching; & $\begin{array}{l}30-100 \mathrm{C}, \quad 0,2 \\
\mathrm{~nm} /{ }^{\circ} \mathrm{C}\end{array}$ & hollow core & intrinsic & {$[145]$} \\
\hline $\begin{array}{l}\text { CdSe-ZnS-TOPO } \\
+\mathrm{Ru}(\mathrm{dpp})\end{array}$ & Sol-gel & $\mathrm{O}_{2}$ & ratiometric detection & $\begin{array}{l}\text { QD as intensity } \\
\text { reference }\end{array}$ & fiber bundle & extrinsic & {$[148]$} \\
\hline $\begin{array}{l}\text { CdSe-ZnS-TOPO } \\
+\mathrm{Ru}(\mathrm{dpp})\end{array}$ & Sol-gel & $\mathrm{O}_{2} \&$ temperature & $\begin{array}{l}\text { luminescence quenching and } \\
\text { bandgap shift; }\end{array}$ & $\begin{array}{l}\text { Simultaneous } \\
\text { detection } \mathrm{O}_{2} \text { and } \\
\text { temperature }\end{array}$ & multimode & extrinsic & {$[147]$} \\
\hline $\begin{array}{l}\text { CdTe-ZnS } \\
+\mathrm{Ru}(\mathrm{dpp})\end{array}$ & Sol-gel & $\mathrm{O}_{2}$ & QD excited by $\mathrm{O}_{2}$ sensing dye. & $\begin{array}{l}\mathrm{O}_{2} \text { sensitivity at } \\
\text { higher } \lambda\end{array}$ & fiber bundle & extrinsic & [148] \\
\hline $\mathrm{CdSe}$ & $\begin{array}{l}\text { solution in } \\
\text { PCF holes }\end{array}$ & Oxidative species & luminescence quenching & $\begin{array}{l}\text { partially } \\
\text { reversible }\end{array}$ & PCF & intrinsic & {$[154]$} \\
\hline Qdot655-ProteinA & Glass plate & IgG & FRET quenching & $\begin{array}{l}\text { fast reagent less } \\
\text { imunodetection }\end{array}$ & fiber bundle & extrinsic & [149] \\
\hline
\end{tabular}

In most of these techniques very bright luminescent patterns could be obtained indicating the preservation of the nanocrystals properties after immobilization. In a recent approach, however, the nanocrystals luminescent emission could be strongly enhanced by coating them over substrates where highly ordered triangular-shaped gold nanopatterns (typical dimensions $200 \mathrm{~nm}$ ) have been fabricated by electron beam lithography [165]. The enhancement is caused by the interaction between the surface plasmon resonances of the metallic structure and the luminescent radiation of the nanocrystals. Surface plasmons are a promising tool for LOC technologies because it enables the control of the quantum yield and the radiation emission pattern of luminescent indicators.

All the described techniques introduce interesting possibilities of using QDs in microarray based configurations. However, sensing applications using such devices is yet to be demonstrated. Nevertheless, an important step towards the realization of QD based micro-arrays biosensors was reported by Sapsford et al.[166]. The authors used glass slides coated with a monolayer of neutravidin as the template. QDs functionalized with maltose binding protein (MBP) and avidin coordinated to their surface were then attached to the glass slides in discrete patterns using an intermediary bridge of biotinylated MBP or antibody linkers. This method allowed to control the surface location and concentration of the QD-protein-based structures. A six-channel patterning PDMS flow cell, was used to define waveguide patterns with the biotin-labeled proteins. Exposure of this biotin-protein patterned waveguides to MBP-QD-avidin then allowed to functionalize the waveguides with the QD probes. Surface FRET events were demonstrated using the fabricated arrays and CCD imaging indicating the feasibility of implementing surface immobilized QD biosensors.

The use of QDs as a gain material in the implementation of laser sources is an highly studied subject. Recently, the possibility of using QD doped cavities as highly sensitive sensors was discussed by Sommers et. al [17]. Distributed feedback (DFB) and 'whispering gallery modes' spherical lasing platforms doped with environmentally responsive nanocrystals can configure extremely sensitive 
biochemical sensors due to the non-linear behavior of the lasing process. In particular, the FRET mechanisms discussed before may be used to introduce analyte induced modulation of the laser gain coefficient.

A striking example of the variety of bio-applications where QDs can introduced significant improvements is the case where $\mathrm{CdSe} / \mathrm{CdS}$ nanocrystal were used to label a cantilever post array to study cellular microforces [167]. Passive bed of nails (BoN) arrays are used to study the forces exerted on the cell surface. The cells are cultured on top of an array of micropilars (Figure 15B) and, since each micropilar behaves like a miniature cantilever, the deformation induced by the forces exerted on the cell walls can be optically monitored. This can be achieved by standard optical microscopy, however, because the refractive index of a cell is close to that of the surrounding aqueous media, it is difficult to identify the borders of the cell under bright field microscopy. Techniques to increase contrast include differential interference contrast microscopy or confocal microcopy. Alternatively, the posts can be labeled with luminescent proteins. However, proteins tend to be dissolved in buffers or ingested by cells. In this context, the use of QDs allowed to obtain a much better contrast and longer experimental observation times without photobleaching, thus improving the tracking of posts.
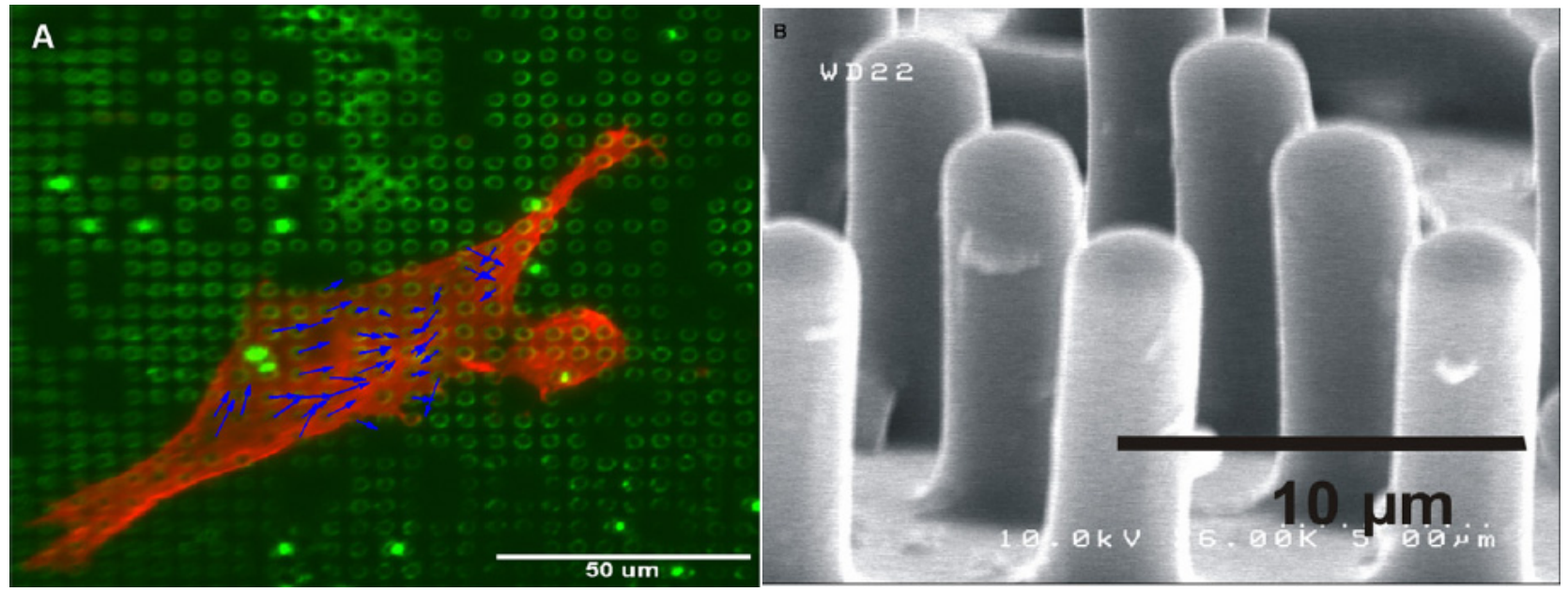

Figure 15. A) Overlay of traction forces on a HASM cell fixed and stained on quantum dot labeled posts. B) SEM of profile of section of BoN $2 \mu \mathrm{m}$ in diameter, $5 \mu \mathrm{m}$ spacing and $7 \mu \mathrm{m}$ tall [167]. Reproduced from Sensors and Actuators A, 136, Addae-Mensah et al., A flexible, quantum dot-labeled cantilever post array for studying cellular microforces, 385-397, Copyright (2007), with permission from Elsevier.

The tops of the posts were coated using a very thin layer of PDMS in which a suspension of quantum dots was previously mixed. The luminescent BoN was then used to obtain the map of the traction forces acting on a human airway smooth muscle (HASM) cell (Figure 15A).

Even though virtually no sensing configuration using QDs in planar platforms has been reported, the fundamental techniques for the implementation of these devices are presently well developed. In this context, it is expected that a burst on the use of QD-based sensing devices in biosensing application is to be expected soon. 


\section{Concluding remarks}

Quantum dots are the basis for well established tools in biomedical imaging applications. In addition, its use in sensing configurations has seen considerable progress with a wide range of chemical and biosensing configurations already demonstrated. However, the application of these sensing principles in practical devices requires the immobilization of functionalized QDs in standard sensing platforms, such as optical fibers and integrated optical chips. Although there have been few reports on the use of QDs to obtain optical fiber or LOC configurations, some important steps were given towards the feasibility of these technologies.

In particular, several versatile immobilization techniques are currently available ranging from LbL to sol-gel or polymer encapsulation that allow to preserve the unique optical properties of QDs. In addition, the use of QD-based sensors while immobilized in such membranes has been reported in many different applications, ranging from multi-ion detection to FRET based immunodetection. Few but representative examples of such sensors were demonstrated at the tip of an optical fiber or in a planar platform. A strong research effort is presently dedicated to QD technology which will contribute for the increasing quality of the available semiconductor nanoparticles, in particular increasing their photostability and limiting their potential toxicity. The same is true for the sensitivity and selectivity of many functionalization strategies.

In this context, a considerable increase in the number of solid platform based QD sensors is to be expected soon. In particular, the convergence of many of the reported techniques will allow to implement high performance instruments: using LbL, the inner surface of microstrucured optical fibers can be functionalized with highly selective QD-antibody conjugates allowing for sensitive FRET detection of different species in simultaneous assays. Coupling such devices with microfluidic capabilities will enable the design of high throughput sensing tools. QDs will also play different roles either as an optical source, as detectors or a sensors. Ultimately, QDs sources themselves can be transformed into extremely sensitive sensing devices. Considering current developments, it is expected that in the near future QD based devices will play an important role in a new generation of nanotechnological instruments.

\section{References and Notes}

1. Liang, S.; Pierce, D. T.; Amiot, C.; Zhao, X. Photoactive nanomaterials for sensing trace analytes in biological samples. Synthesis and Reactivity in Inorganic Metal-Organic and Nano-Metal Chemistry 2005, 35(9), 661-668.

2. Ozkan, M. Quantum dots and other nanoparticles: what can they offer to drug discovery? Drug Discovery Today 2004, 9(24), 1065-1071.

3. Willner, I.; Basnar, B.; Willner, B. Nanoparticle-enzyme hybrid systems for nanobiotechnology. Febs Journal 2007, 274(2), 302-309.

4. Penn, S.G.; He, L.; Natan, M.J. Nanoparticles for bioanalysis. Current Opinion in Chemical Biology 2003, 7(5), 609-615. 
5. Kurner, J.M.; Klimant, I.; Krause, C.; Pringsheim, E.; Wolfbeis, O.S. A New Type of Phosphorescent Nanospheres for Use in Advanced Time-Resolved Multiplexed Bioassays. Analytical Biochemistry 2001, 297, 32-41.

6. Teolato, P. et al. Silica nanoparticles for fluorescence sensing of Zn-II: Exploring the covalent strategy. Chemistry-a European Journal 2007, 13(8), 2238-2245.

7. Bruchez Jr., M.B. et al. Semiconductor nanocrystals as fluorescent biological labels. Science 1998, 281, 2013-2016.

8. Mattoussi, H. et al. Luminescent quantum dot-bioconjugates in immunoassays, FRET, biosensing, and imaging applications. JALA, 2004, 9, 28-32.

9. Zhang, Y. et al. Using cadmium telluride quantum dots as a proton flux sensor and applying to detect H9 avian influenza virus. Analytical Biochemistry 2007, 364(2), 122-127.

10. Trindade, T.; O'Brien, P.; Pickett, N.L. Nanocrystalline semiconductors: synthesis, properties, and perspectives. Chemistry of Materials 2001, 13(11), 3843-3858.

11. Murphy, C.J. Optical sensing with quantum dots. Analytical Chemistry 2002, 74, 520A-526A.

12. Jaiswal, J.K.; Simon, S.M. Potentials and pitfalls of fluorescent quantum dots for biological imaging. Trends in Cell Biology 2004, 14(9), 497-504.

13. Riegler, J.; Nann, T. Application of luminescent nanocrystals as labels for biological molecules. Anal Bioanal Chem 2004, 379, 913-919.

14. Costa-Fernandez, J.M.; Pereiro, R.; Sanz-Medel, A. The use of luminescent quantum dots for optical sensing. Trac-Trends in Analytical Chemistry 2006, 25(3), 207-218.

15. Clapp, A.R.; Medintz, I.L.; Mattoussi, H. Forster resonance energy transfer investigations using quantum-dot fluorophores. Chemphyschem 2006, 7(1), 47-57.

16. Wang, X.; Ruedas-Rama, M.J.; Hall, E.A.H. The Emerging Use of Quantum Dots in Analysis. Analytical Letters 2007, 40, 1497-1520.

17. Somers, R.C.; Bawendi, M.G.; Nocera, D.G. CdSe nanocrystal based chem-/bio- sensors. Chem. Soc. Rev. 2007, 36, 579-591.

18. Klimov, V.I. Nanocrystal quantum dots. Los Alamos Science 2003, 28, 214-220.

19. Grundmann, M. The present status of quantum dot lasers. Physica E 2000, 5, 167-184.

20. Skolnick, M.S.; Mowbray, D.J. Self-assembled semiconductor quantum dots: Fundamental physics and device applications. Annual Review of Materials Research 2004, 34, 181-218.

21. Scherer, A.; Craighead, H.G. Fabrication of small laterally patterned multiple quantum-wells. Applied Physics Letters 1986, 49(19), 1284-1286.

22. Fukui, T. et al. GaAs tetrahedral quantum dot structures fabricated using selective area metalorganic chemical vapor-deposition. Applied Physics Letters 1991, 58(18), 2018-2020.

23. Gaponenko, S.V. Optical properties of semiconductor nanocrystals; Cambridge Studies in Modern Optics; Ed. P. L. Knight and A. Miller.: Cambridge: Cambridge University Press; Vol. 23. 1998

24. Bawendi, M.G.; Steigerwald, M.L.; Brus, L.E. The quantum mechanics of larger semiconductor clusters ("quantum dots"). Annual Review of Physical Chemistry 1990, 41, 477-496.

25. Hines, M.A.; Guyot-Sionnest, P. Synthesis and characterization of strongly luminescing ZnScapped CdSe nanocrystals. J. Phys. Chem. 1996, 100, 468-471. 
26. Dabbousi, B.O. et al. (CdSe)ZnS core-shell quantum dots: Synthesis and characterization of a size series of highly luminescent nanocrystallites. Journal of Physical Chemistry B 1997, 101(46), 9463-9475.

27. Xie, R. et al. Synthesis and Characterization of Highly Luminescent CdSe-Core CdS/Zn0.5Cd0.5S/ZnS Multishell Nanocrystals. J. Am. Chem. Soc. 2005, 127, 7480-7488.

28. Korsunska, N.E. et al. Reversible and non-reversible photo-enhanced luminescence in CdSe/ZnS quantum dots. Semiconductor Science and Technology 2005, 20(8), 876-881.

29. Zhelev, Z. et al. Enhancement of the photoluminescence of CdSe quantum dots during long-term UV-irradiation: privilege or fault in life science research? J Photochem. Photobiol. B 2004, 75(12), 99-105.

30. Verma, P.; Irmer, G.; Monecke, J. Laser power dependence of the photoluminescence from CdSxSe1-x nanoparticles in glass. Journal of Physics-Condensed Matter 2000, 12(6), 1097-1110.

31. Brus, L.E. A Simple-Model for the Ionization-Potential, Electron-Affinity, and Aqueous Redox Potentials of Small Semiconductor Crystallites. Journal of Chemical Physics 1983, 79(11), 55665571.

32. Murray, C.B.; Kagan, C.R.; Bawendi, M.G. Synthesis and characterization of monodisperse nanocrystals and close-packed nanocrystal assemblies. Annual Review of Materials Science 2000, 30, 545-610.

33. Murray, C.B.; Norris, D.J.; Bawendi, M.G. Synthesis and Characterization of Nearly Monodisperse Cde $(\mathrm{E}=\mathrm{S}$, Se, Te) Semiconductor Nanocrystallites. Journal of the American Chemical Society 1993, 115(19), 8706-8715.

34. Cozzoli, P.D. et al. Shape and phase control of colloidal ZnSe nanocrystals. Chemistry of Materials 2005, 17(6), 1296-1306.

35. Trindade, T.; OBrien, P. A single source approach to the synthesis of CdSe nanocrystallites. Advanced Materials 1996, 8(2), 161-163.

36. Trindade, T.; OBrien, P.; Zhang, X.M. Synthesis of CdS and CdSe nanocrystallites using a novel single-molecule precursors approach. Chemistry of Materials 1997, 9(2), 523-530.

37. Nair, P.S. et al. Cadmium ethylxanthate: A novel single-source precursor for the preparation of CdS nanoparticles. Journal of Materials Chemistry 2002, 12(9), 2722-2725.

38. Malik, M.A.; O'Brien, P.; Revaprasadu, N. A simple route to the synthesis of core/shell nanoparticles of chalcogenides. Chemistry of Materials 2002, 14(5), 2004-2010.

39. Murcia, M.J. et al. Facile sonochemical synthesis of highly luminescent ZnS-shelled CdSe quantum dots. Chemistry of Materials 2006, 18(9), 2219-2225.

40. Peng, Z.A.; Peng, X.G. Formation of high-quality CdTe, CdSe, and CdS nanocrystals using CdO as precursor. Journal of the American Chemical Society 2001, 123(1), 183-184.

41. Peng, X.G. Green chemical approaches toward high-quality semiconductor nanocrystals. Chemistry-a European Journal 2002, 8(2), 335-339.

42. Rogach, A. et al. Colloidally prepared HgTe nanocrystals with strong room-temperature infrared luminescence. Advanced Materials 1999, 11(7), 552-555.

43. Vossmeyer, T. et al. Cds Nanoclusters - Synthesis, Characterization, Size-Dependent Oscillator Strength, Temperature Shift of the Excitonic-Transition Energy, and Reversible Absorbency Shift. Journal of Physical Chemistry 1994, 98(31), 7665-7673. 
44. Rogach, A.L. et al. Synthesis and characterization of a size series of extremely small thiolstabilized CdSe nanocrystals. Journal of Physical Chemistry B 1999, 103(16), 3065-3069.

45. Rogach, A.L. et al. Synthesis and characterization of thiol-stabilized CdTe nanocrystals. Berichte Der Bunsen-Gesellschaft-Physical Chemistry Chemical Physics 1996, 100(11), 1772-1778.

46. Rogach, A.L. et al. Synthesis, morphology and optical properties of thiol-stabilized CdTe nanoclusters in aqueous solution. Berichte Der Bunsen-Gesellschaft-Physical Chemistry Chemical Physics 1997, 101(11), 1668-1670.

47. Gao, M.Y. et al. Strongly photoluminescent CdTe nanocrystals by proper surface modification. Journal of Physical Chemistry B 1998, 102(43), 8360-8363.

48. Green, M. et al. A facile route to CdTe nanoparticles and their use in bio-labelling. Journal of Materials Chemistry 2007, 17(19), 1989-1994.

49. Aldana, J.; Wang, Y.A., Peng, X.G. Photochemical instability of CdSe nanocrystals coated by hydrophilic thiols. Journal of the American Chemical Society 2001, 123(36), 8844-8850.

50. Liu, T.C. et al. Temperature-dependent photoluminescence of water-soluble quantum dots for a bioprobe. Analytica Chimica Acta 2006, 559(1), 120-123.

51. Liu, Y.S. et al. pH-sensitive photoluminescence of $\mathrm{CdSe} / \mathrm{ZnSe} / \mathrm{ZnS}$ quantum dots in human ovarian cancer cells. Journal of Physical Chemistry C 2007, 111(7), 2872-2878.

52. Huang, C.P. et al. Plate-based biochemical assay using quantum dots as a fluorescent labeling agent. Sensors and Actuators B-Chemical 2005, 108(1-2), 713-720.

53. Mattoussi, H. et al. Self-assembly of CdSe-ZnS quantum dot bioconjugates using an engineered recombinant protein. Journal of the American Chemical Society 2000, 122(49), 12142-12150.

54. Uyeda, H.T. et al. Synthesis of compact multidentate ligands to prepare stable hydrophilic quantum dot fluorophores. Journal of the American Chemical Society 2005, 127(11), 3870-3878.

55. Kim, S.; Bawendi, M.G. Oligomeric Ligands for luminescent and stable nanocrystal quantum dots. Journal of the American Chemical Society 2003, 125(48), 14652-14653.

56. Guo, W.H. et al. Luminescent $\mathrm{CdSe} / \mathrm{CdS}$ core/shell nanocrystals in dendron boxes: Superior chemical, photochemical and thermal stability. Journal of the American Chemical Society 2003, 125(13), 3901-3909.

57. Guo, W.Z. et al. Conjugation chemistry and bioapplications of semiconductor box nanocrystals prepared via dendrimer bridging. Chemistry of Materials 2003, 15(16), 3125-3133.

58. Gerion, D. et al. Synthesis and properties of biocompatible water-soluble silica-coated CdSe/ZnS semiconductor quantum dots. Journal of Physical Chemistry B 2001, 105(37), 8861-8871.

59. Iler, R.K. The Chemistry of Silica: Solubility, Polymerization, Colloid and Surface Properties and Biochemistry of Silica.; John Wiley \& Sons: New York; 1979.

60. Wu, X.Y. et al. Immunofluorescent labeling of cancer marker Her2 and other cellular targets with semiconductor quantum dots. Nature Biotechnology 2003, 21(1), 41-46.

61. Pellegrino, T. et al. Hydrophobic nanocrystals coated with an amphiphilic polymer shell: A general route to water soluble nanocrystals. Nano Letters 2004, 4(4), 703-707.

62. Gao, X.H. et al. In vivo cancer targeting and imaging with semiconductor quantum dots. Nature Biotechnology 2004, 22(8), 969-976.

63. Dubertret, B. et al. In vivo imaging of quantum dots encapsulated in phospholipid micelles. Science 2002, 298(5599), 1759-1762. 
64. Mattheakis, L.C. et al. Optical coding of mammalian cells using semiconductor quantum dots. Analytical Biochemistry 2004, 327(2), 200-208.

65. Ballou, B. et al. Noninvasive imaging of quantum dots in mice. Bioconjugate Chemistry 2004, 15(1), 79-86.

66. Jin, T. et al. Calixarene-coated water-soluble CdSe-ZnS semiconductor quantum dots that are highly fluorescent and stable in aqueous solution. Chemical Communications 2005, 22, 28292831.

67. Jin, T. et al. Amphiphilic p-sulfonatocalix[4]arene-coated $\mathrm{CdSe} / \mathrm{ZnS}$ quantum dots for the optical detection of the neurotransmitter acetylcholine. Chemical Communications 2005, 34, 4300-4302.

68. Liu, J.A. et al. Use of ester-terminated polyamidoamine dendrimers for stabilizing quantum dots in aqueous solutions. Small 2006, 2(8-9), 999-1002.

69. Erdem, B. et al. Encapsulation of inorganic particles via miniemulsion polymerization. III. Characterization of encapsulation. Journal of Polymer Science Part a-Polymer Chemistry 2000, $38(24), 4441-4450$.

70. Erdem, B. et al. Encapsulation of inorganic particles via miniemulsion polymerization. II. Preparation and characterization of styrene miniemulsion droplets containing $\mathrm{TiO} 2$ particles. Journal of Polymer Science Part a-Polymer Chemistry 2000, 38(24), 4431-4440.

71. Erdem, B. et al. Encapsulation of inorganic particles via miniemulsion polymerization. I. Dispersion of titanium dioxide particles in organic media using OLOA 370 as stabilizer. Journal of Polymer Science Part a-Polymer Chemistry 2000, 38(24), 4419-4430.

72. Tiarks, F.; Landfester, K.; Antonietti, M. Silica nanoparticles as surfactants and fillers for latexes made by miniemulsion polymerization. Langmuir 2001, 17(19), 5775-5780.

73. Tiarks, F.; Landfester, K.; Anonietti, M. Encapsulation of carbon black by miniemulsion polymerization. Macromolecular Chemistry and Physics 2001, 202(1), 51-60.

74. Esteves, A.C.C. et al. Polymer encapsulation of $\mathrm{CdE}(\mathrm{E}=\mathrm{S}, \mathrm{Se})$ quantum dot ensembles via insitu radical polymerization in miniemulsion. Journal of Nanoscience and Nanotechnology 2005, 5(5), 766-771.

75. Joumaa, N. et al. Synthesis of quantum dot-tagged submicrometer polystyrene particles by miniemulsion polymerization. Langmuir 2006, 22(4), 1810-1816.

76. Martins, M.A. et al. Biofunctionalized ferromagnetic CoPt3/polymer nanocomposites. Nanotechnology 2007, 18(21), 5609-5615.

77. Fleischhaker, F.; Zentel, R. Photonic crystals from core-shell colloids with incorporated highly fluorescent quantum dots. Chemistry of Materials 2005, 17(6), 1346-1351.

78. Peres, M. et al. A green-emitting CdSe/poly(butyl acrylate) nanocomposite. Nanotechnology 2005, 16(9), 1969-1973.

79. Zhu, M.Q. et al. Surface modification and functionalization of semiconductor quantum dots through reactive coating of silanes in toluene. Journal of Materials Chemistry 2007, 17(8), 800805.

80. Ohno, K. et al. Fabrication of ordered arrays of gold nanoparticles coated with high-density polymer brushes. Angewandte Chemie-International Edition 2003, 42(24), 2751-2754.

81. Bao, Z.Y.; Bruening, M.L.; Baker, G.L. Rapid growth of polymer brushes from immobilized initiators. Journal of the American Chemical Society 2006, 128(28), 9056-9060. 
82. Ohno, K. et al. Synthesis of monodisperse silica particles coated with well-defined, high-density polymer brushes by surface-initiated atom transfer radical polymerization. Macromolecules $\mathbf{2 0 0 5}$, 38(6), 2137-2142.

83. Zhao, H.Y.; Kang, X.L.; Liu, L. Comb-coil polymer brushes on the surface of silica nanoparticles. Macromolecules 2005, 38(26), 10619-10622.

84. Marutani, E. et al. Surface-initiated atom transfer radical polymerization of methyl methacrylate on magnetite nanoparticles. Polymer 2004, 45(7), 2231-2235.

85. Skaff, H.; Emrick, T. Reversible addition fragmentation chain transfer (RAFT) polymerization from unprotected cadmium selenide nanoparticles. Angewandte Chemie-International Edition 2004, 43(40), 5383-5386.

86. Sill, K.; Emrick, T. Nitroxide-mediated radical polymerization from CdSe nanoparticles. Chemistry of Materials 2004, 16(7), 1240-1243.

87. Esteves, A.C.C. et al. Polymer grafting from Cds quantum dots via AGET ATRP in miniemulsion. Small 2007, 3(7), 1230-1236.

88. Wu, Y.-h.; Arai, K.; Yao, T. Temperature dependence of the photoluminescence of $\mathrm{ZnSe} / \mathrm{ZnS}$ quantum-dot structures. Physical Review B 1996, 53(16), R 10485 - R 10488.

89. Labeau, O.; Tamarat, P.; Lounis, B. Temperature dependence of the luminescence lifetime of single CdSe/ZnS quantum dots. Physical Review Letters 2003, 90(25), 7404-7407.

90. Biju, V. et al. Temperature-sensitive photoluminescence of CdSe quantum dot clusters. Journal of Physical Chemistry B 2005, 109(29), 13899-13905.

91. Walker, G.W. et al. Quantum-dot optical temperature probes. Applied Physics Letters 2003, 83(17), 3555-3557.

92. Chen, Y.; Rosenzweig, Z. Luminescent CdS quantum dots as selective ion probes. Analytical Chemistry 2002, 74(19), 5132-5138.

93. Xie, H.-Y. et al. Luminescent CdSe-ZnS quantum dots as selective $\mathrm{Cu} 2+$ probe. Spectrochimica Acta Part A 2004, 60, 2527-2530.

94. Jin, W.J. et al. Surface-modified CdSe quantum dots as luminescent probes for cyanide determination. Analytica Chimica Acta 2004, 522, 1-8.

95. Shi, G.H. et al. Fluorescence quenching of CdSe quantum dots by nitroaromatic explosives and their relative compounds. Spectrochimica Acta Part A 2007.

96. Albert, K.e.J. Field-Deployable Sniffer for 2,4-Dinitrotoluene Detection. Environ. Sci. Technol. 2001, 35, 3193-3200.

97. Nazzal, A.Y. et al. Photoactivated CdSe Nanocrystals as Nanosensors for Gases. Nano Lett. 2003, 3(6), 819-822.

98. Potyrailo, R.A.; Leach, A.M. Selective gas nanosensors with multisize CdSe nanocrystal/polymer composite films and dynamic pattern recognition. Applied Physics Letters 2006, 88, 134110.

99. Tomasulo, M. et al. pH-Sensitive Ligand for Luminescent Quantum Dots. Langmuir 2006, 22, 10284-10290.

100. Snee, P.T. et al. A Ratiometric CdSe/ZnS Nanocrystal pH Sensor. J. Am. Chem. Soc. 2006, 128, 13320-13321.

101. Ruedas-Rama, M.J.; Wang, X.; Hall, E.A.H. A multi-ion particle sensor. Chem. Commun. 2007, 1544-1546. 
102. Xu, C.; Bakker, E. Multicolor Quantum Dot Encoding for Polymeric Particle-Based Optical Ion Sensors. Anal. Chem. 2007, 79, 3716-3723.

103. Lin, C.I. et al. Molecularly imprinted polymeric film on semiconductor nanoparticles: analyte detection by quantum dot photoluminescence. Journal of Chromatography A 2004, 1027, 259262.

104. Alivisatos, P. The use of nanocrystals in biological detection. Nature Biotechnology 2004, 22(1), 47-52.

105. Evident Technologies. cited 2007; Available from: www.evidenttech.com.

106. Quantum Dot Corporation. cited 2007; Available from: www.qdots.com.

107. Chan, W.C.W.; Nie, S. Quantum dot bioconjugates for ultrasensitive nonisotopic detection. Science 1998, 281, 2016-2018.

108. Sark, W.G.J.H.M.v., et al., Blueing, bleaching, and blinking of single CdSe/ZnS quantum dots. Chemphyschem, 2002. 3(10): 871-879.

109. Jaiswal, J.K. et al. Long-term multiple color imaging of live cells using quantum dot bioconjugates. Nature Biotechnology 2003, 21(1), 47-51.

110. Gao, X. et al. In vivo molecular and cellular imaging with quantum dots. Current Opinion in Biotechnology 2005, 16, 63-72.

111. Smith, A.M. et al. Multicolor quantum dots for molecular diagnostics of cancer. Exopert Rev. Mol. Diagn. 2006.

112. Smith, J.D. et al. The use of quantum dots for analysis of chick CAM vasculature. Microvascular Research 2007, 73, 75-83.

113. Murphy, C.; Coffer, J. Quantum dots: a primer. Appl. Spectrosc. 2002, 56(1), 16A-27A.

114. Liang, J. et al. Study on DNA damage induced by CdSe quantum dots using nucleic acid molecular "light switches" as probe. Talanta 2007, 71, 1675-1678.

115. Guo, G. et al. Probing the cytotoxicity of CdSe quantum dots with surface modification. Materials Letters 2007, 61, 1641-1644.

116. Mattoussi, H. et al. Self-assembly of CdSe-ZnS quantum dot bioconjugates using an engineered recombinant protein. Journal of the American Chemical Society 2000, 122(49), 12142-12150.

117. Clapp, A.R. et al. Fluorescence resonance energy transfer between quantum dot donors and dyelabeled protein acceptors. Journal of the American Chemical Society 2004, 126(1), 301-310.

118. Liu, Z.D. et al. Light scattering sensing detection of pathogens based on the molecular recognition of immunoglobulin with cell wall-associated protein A. Analytica Chimica Acta 2007, 599, 279286.

119. Yu, Y. et al. Synthesis of functionalized CdTe/CdS QDs for spectrofluorimetric detection of BSA. Spectrochim. Acta Part A: Mol. Biomol. Spectrosc. 2007. doi:10.1016/j.saa.2007.02.016.

120. Stsiapura, V. et al. Functionalized nanocrystal-tagged fluorescent polymer beads: synthesis, physicochemical characterization and immunolableing application. Analytical Biochhemistry 2004, 334, 257-265.

121. Wang, H.-Q. et al. Multicolor encoding of polystyrene microbeads with CdSe-ZnS quantum dots and its application in immunoassay. Jornal of Colloid and Interface Science 2007, doi:10.1016/j.jcis 2007.08.065. 
122. Cordes, D.B.; Gamsey, S.; Singaram, B. Fluorescent Quantum Dots with Boronic Acid Substituted Viologens To Sense Glucose in Aqueous Solution. Angew. Chem. Int. Ed. 2006, 45, $3829-3832$.

123. Huang, C.-P. et al. A new approach for quantitative determination of glucose by using CdSe/ZnS quantum dots. Sens. Actuators B: Chem 2007, doi:10.1016/j.snb.2007.08.021.

124. Holst, G.; Mizaikoff, B. Fiber Optic Sensors for Environmental Applications; In Handbook of Optical Fibre Sensing Technology; John Wiley \& Sons, LTD. 2002, pp. 729-755.

125. Grattan, K.T.V.; Sun, T. Fiber optic sensor technology: an overview. Sensors and Actuators aPhysical 2000, 82(1-3), 40-61.

126. Wolfbeis, O.S. Fiber Optic Chemical Sensors and Biosensors. Anal. Chem. 2002, 72, 81R-89R.

127. Wolfbeis, O.S. Fiber Optic Chemical Sensors and Biosensors. Anal. Chem. 2004, 76, 3269-3284.

128. Butler, T.M.; MacCraith, B.D.; McDonagh, C. Leaching in sol-gel-derived silica films for optical pH sensing. Journal of Non-Crystalline Solids 1998, 224, 249-258.

129. Hartmann, P.; Leiner, M.J.P.; Kohlbacher, P. Photobleaching of ruthenium complex in polymers used for oxygen optodes and its inhibition by singlet oxygen quenchers. Sensors and Actuators $B$ 1998, 51, 196-202.

130. Costa, V.C.; Shen, Y.; Bray, K.L. Luminescence properties of nanocrystalline CdS and CdS:Mn2+ doped silica-type glasses. Journal of Non-Crystalline Solids 2002, 304, 217-223.

131. Litran, R. et al. Confinement of CdS Nanocrystals in a Sonogel Matrix. Journal of Sol-Gel Science and Technology 1997, 8, 275-283

132. Wang, Y. et al. Optical responses of ZnSe quantum dots in silica gel glasses. Journal of Crystal Growth 2004, 268, 580-584.

133. Arachchige, I.U.; Brock, S.L. Sol-Gel Methods for the Assembly of Metal Chalcogenide Quantum Dots. Acc. Chem. Res. 2007, 40, 801-809.

134. Reisfeld, R.; Saraidarov, T. Innovative materials based on sol-gel technology. Optical Materials 2006, 28, 64-70.

135. Bullen, C. et al. Incorporation of a highly luminescent semiconductor quantum dot in $\mathrm{ZrO} 2-\mathrm{SiO} 2$ hybrid sol-gel glass film. J. Mater. Chem. 2004, 14, 1112-1116.

136. Ferreira, P.M.S. et al. Langmuir-Blodgett manipulation of capped cadmium sulfide quantum dots. Thin Solid Films 2001, 389, 272-277.

137. Crisp, M.T.; Kotov, N.A. Preparation of Nanoparticle Coatings on Surfaces of Complex Geometry. Nano Lett. 2003, 3(2), 174-177.

138. Barmenkov, Y.O.; Starodumov, A.N.; Lipovskii, A.A. Temperature fiber sensor based on semiconductor nanocrystallite-doped phosphate glasses. Applied Physics Letters 1998, 73, 4.

139. Jorge, P.A.S. et al. Luminescence-based optical fiber chemical sensors. Fiber and Integrated Optics 2005, 24(3-4), 201-225.

140. Jorge, P.A.S. et al. Quantum dots as self-referenced optical fibre temperature probes for luminescent chemical sensors. Measurement Science \& Technology 2006, 17(5), 1032-1038.

141. Benrashid, R.; Velasco, P. High performance sol-gel spin-on glass materials; Waveguide Solutions: Charlotte, NC; 2005: p. 27.

142. Odonnell, K.P.; Chen, X. Temperature-dependence of semiconductor band-gaps. Applied Physics Letters 1991, 58(25), 2924-2926. 
143. Valerini, D. et al. Temperature dependence of the photoluminescence properties of colloidal $\mathrm{CdSe} / \mathrm{ZnS}$ core/shell quantum dots embedded in a polystyrene matrix. Phys. Rev. B 2005, 71, 235409.

144. deBastida, G. et al. Quantum dots based optical fiber temperature sensors fabricated by Layer-byLayer. IEEE Sensors Journal, 2006.

145. Bravo, J. et al. Fiber Optic temperature sensor depositing quantum dots inside hollow core fibers using layer by layer technique. In EWOFS 2007. Napoli: SPIE. 6619: 661919-1; 2007.

146. Ruan, H. et al. Self assembled optical detectors for optical fiber sensors. In EWOFS 2007. Napoli: SPIE. 6619: 66192W-1; 2007.

147. Jorge, P.A.S. et al. Simultaneous determination of oxygen and temperature using quantum dots and a ruthenium complex. In EWOFS 2007. Napoli: SPIE. 6619: 66191Y; 2007.

148. Jorge, P.A.S. et al. Applications of quantum dots in optical fiber luminescent oxygen sensors. Applied Optics 2006, 45(16), 3760-3767.

149. Aoyagi, S.; Kudo, M. Development of fluorescence change-based, reagent-less optic immunosensor. Biosensors and Bioelectronics 2005, 20, 1680-1684.

150. Meissner, K.E.; Holton, C.; Spillmann Jr., W.B. Optical characterization of quantum dots entrained in microstructured optical fibers. Physica E 2005, 26, 377-381.

151. Finlayson, C.E. Comment on "Optical characterization of quantum dots entrained in microstructured optical fibers"' Physica E 26 (2005) 377-381. Physica E 2006, 31, 107-108.

152. Meissner, K.E.; Holton, C.; Spillmann Jr., W.B. Response to comment on "Optical characterization of quantum dots entrained in microstructured optical fibers" Physica E 2006, 31, 109-110.

153. Yu, H.C.Y. et al. Quantum dot and silica nanoparticle doped polymer optical fibers. Optics Express 2007, 15(16), 9989-9994.

154. Galian, R.E.; Laferriere, M.; Scaiano, J.C. Doping of photonic crystal fibers with fluorescent probes: possible functional materials for optrode sensors. J. Mater. Chem. 2006, 16, 1697-1701.

155. Rindorf, L. et al. Towards biochip using microstructured optical fiber sensors. Anal. Bioanal. Chem. 2006, 385, 1370-1375.

156. Hassani, A.; Skorobogatiy, M. Design of the Microstructured Optical Fiber-based Surface Plasmon Resonance sensors with enhanced microfluidics. Optics Express 2006, 14(24 ), 11616.

157. Craighead, H. Future lab-on-a-chip technologies for interrogating individual molecules. Nature 2006, 442(7101), 387-393.

158. Dittrich, P.S.; Manz, A. Lab-on-a-chip: microfluidics in drug discovery. Nature Reviews Drug Discovery 2006, 5(3), 210-218.

159. Loonberg, M.; Carlsson, J. Lab-on-a-chip technology for determination of protein isoform profiles. Journal of Chromatography A 2006, 1127(1-2), 175-182.

160. Riegger, L. et al. Read-out concepts for multiplexed bead-based fluorescence immunoassays on centrifugal microfluidic platforms. Sensors and Actuators A 2006, 126, 455-462.

161. Vossmeyer, T. et al. Combinatorial approaches toward patterning nanocrystals. Journal of Applied Physics 1998, 84(7), 3664-3670.

162. Meldrun, A. et al. Micropixelated Luminescent nanocrystal arrays synthesized by ion implantation. Advanced Materials 2004, 16(1), 31-34. 
163. Chen, C.-C. et al. Self-Assembly of Monolayers of Cadmium Selenide Nanocrystals with Dual Color Emission. Langmuir 1999, 15, 6845-6850.

164. Bertino, M.F. et al. Patterning porous matrices and planar substrates with quantum dots. $J$ Sol-Gel Sci Techn 2006, 39, 299-306.

165. Pompa, P.P. et al. Fluorescence enhancement in colloidal semiconductor nanocrystals by metallic nanopatterns. Sensors and Actuators B 2007, 126, 187-192.

166. Sapsford, K.E. et al. Surface-Immobilized Self-Assembled Protein-Based Quantum Dot Nanoassemblies. Langmuir 2004, 20, 7720-7728.

167. Addae-Mensah, K.A. et al. A flexible, quantum dot-labeled cantilever post array for studying cellular microforces. Sensors and Actuators A 2007, 136, 385-397.

(C) 2007 by MDPI (http://www.mdpi.org). Reproduction is permitted for noncommercial purposes. 